\title{
Impact of Instrumental Systematic Contamination on the Lensing Mass Reconstruction using the CMB Polarization
}

\author{
Meng Su${ }^{1}$ 团 Amit P.S. Yadav ${ }^{1}$, and Matias Zaldarriaga ${ }^{1,2}$ \\ ${ }^{1}$ Harvard-Smithsonian Center for Astrophysics, 60 Garden St., Cambridge, MA 02138, USA and \\ ${ }^{2}$ Jefferson Laboratory of Physics, Harvard University, Cambridge, MA 02138, USA
}

\begin{abstract}
In this paper, we study the effects of instrumental systematics on the reconstruction of the deflection angle power spectrum from weak lensing of Cosmic Microwave Background (CMB) temperature and polarization observations. We consider 7 types of effects which are related to known instrumental systematics: calibration, rotation, pointing, spin-flip, monopole leakage, dipole leakage and quadrupole leakage. These effects can be characterized by 11 distortion fields. Each of these systematic effects can mimic the effective projected matter power spectrum and hence contaminate the lensing reconstruction. To demonstrate the effect of these instrumental systematics on CMB lensing measurements, we consider two types of experiments, one with a detector noise level for polarization of $9.6 \mu \mathrm{K}$-arcmin and FWHM of 8.0', typical of upcoming ground and balloon-based $\mathrm{CMB}$ experiments, and a CMBPol-like instrument with a detector noise level for polarization of $2.0 \mu \mathrm{K}$-arcmin and FWHM of $4.0^{\prime}$, typical of future space-based CMB experiments. For each systematics field, we consider various choices of coherence scale $\alpha_{s}$, starting from $\alpha_{s}=10^{\prime}$ to the maximum of $\alpha_{s}=120^{\prime}$. Among all the 11 systematic parameters, rotation $\omega$, and monopole leakage $\gamma_{a} \& \gamma_{b}$ place the most stringent requirements, while quadrupole leakage $q$, pointing error $p_{a}$ and $p_{b}$, and calibration $a$ are among the least demanding. The requirements from lensing extraction are about 1-2 orders of magnitude less stringent than the requirements to measure the primordial B-modes with inflationary energy scale of $1.0 \times 10^{16} \mathrm{GeV}$. On the other hand the requirements for lensing reconstruction are comparable or even more stringent for some systematic parameters than the requirements to detect primordial B-modes with inflationary scale $E_{i}=3.0 \times 10^{16} \mathrm{GeV}$.
\end{abstract}

PACS numbers:

\section{INTRODUCTION}

Observations of the temperature anisotropies of the Cosmic Microwave Background (CMB) have been a major tool to constrain cosmological parameters. The polarization data of the CMB can help us to extract additional information beyond the temperature information [1, 2]. The next generation of CMB observations will focus on the precise measurement of polarization of the $\mathrm{CMB}$, especially the so called B-mode polarization, which is at least two orders of magnitude smaller than the temperature anisotropy signal. In contrast to E-mode polarization, which can be generated by scalar or tensor perturbations in the early universe, the primordial B-modes are generated only by tensor perturbations [3, 4]. However even in the absence of primordial B-modes, subsequent gravitational lensing by the large scale structure of the Universe converts E-mode polarization to B-mode polarization [5, 6, 7, 8]. Although the amplitude of the primordial gravitational wave signal is uncertain by many orders magnitude and might not be detectable by the next generation of polarization experiments, the lensing B-mode signal is a guaranteed prediction of the current cosmological model. In addition, the B-mode lensing signal will help to break degeneracies between cosmological parameters [9, 10, 11, 12, 13, 14].

The weak gravitational lensing of CMB anisotropies provides a unique opportunity to map the matter distribution of the universe. The non-Gaussian higher order correlations in the CMB generated by the weak lensing can be used to reconstruct the mass distribution of the intervening large-scale structure. The principle is easy to understand. The CMB photons are remapped by gravitational lensing which introduces correlations between different angular moments. One way of extracting the lensing information is to use a quadratic combination of the CMB multipoles to define an estimator for the projected gravitational potential [15, 16, 17, 18, 19, 20, 21, 22].

Although the B-mode polarization observations are currently still noise dominated, the next generation of CMB polarization instruments has the sensitivity to make first detections, at least of the lensing induced B-mode signal. However, there are several challenges for CMB lensing detection, mainly coming from astrophysical foregrounds and instrumental systematics. It is important to estimate and control those spurious signals as well as possible when analyzing upcoming CMB data. These challenges will have to be overcome in order to probe the physics of the early universe through $B$-mode polarization or to infer the projected large scale matter distribution from E/B polarization.

*Electronic address: mengsu@cfa.harvard.edu 
Lensing studies can be considered as secondary science for an experiment devoted to B-mode detection. Impact of instrumental systematics on the projected matter power spectrum is helpful to both instrument design and future data forecast. On the other hand, lensing induced $B$-mode polarization is a cosmological contaminant for the detection of primordial $B$-modes, a systemic study of instrumental systematics for lensing reconstruction may well be required of such an experiment to delense the observed CMB fields [23, 24, 25, 26, 27, 28, 29, 30, 31].

In the literature, instrumental systematics have been discussed extensively [32, 33, 34, 35, 36, 37, 38, 39]. The main goal of this paper is to illustrates the effects of instrumental systematics and systematically study the impact on the mass reconstruction process for upcoming CMB experiments [24, 25, 26, 27, 28, 29, 30]. To calculate the effects of instrumental systematics on the projected matter power spectrum, we make use of the quadratic estimator to reconstruct the projected gravitational potential [15], and classify and parameterize the systematics following [32]. We divide polarization contaminations into two categories: those which are associated with a transfer between the polarization state of the incoming radiation (from detection system), including calibration and rotation, spin-flip coupling and monopole leakage errors, and those which are associated with CMB anisotropy induced by the finite resolution or beam of the telescope. For the polarization transfer systematics, we only concern ourselves with polarization transfer in a single, perfectly known, direction on the sky. However, in reality, every experiment necessarily has finite resolution and this therefore is an additional class of contamination associated with the resolution or beam of the experiment. We refer the reader to [32] for a detailed discussion of the parametrization of the systematic errors we use in this paper. We calculate the unlensed and lensed CMB power spectrum using CMBFAST [40]. In the calculation, we have assumed a flat $\Lambda C D M$ cosmology with following cosmological parameter values: $\Omega_{b}=0.045, \Omega_{c}=0.23, H_{0}=70.5, n_{s}=$ $0.96, n_{t}=0.0, \tau=0.08$.

This paper is organized as follows: In Sec. II we review the basic lensing formalism, define our notation, and introduce the quadratic estimator of the lensing potential reconstruction which we later use to explore the effects of instrumental systematics. In Sec. [III we first consider a relatively simple case of the calibration systematics on the temperature estimator of lensing reconstruction. Then we consider EB estimator as an example, studying 7 types of instrumental systematic effects on lensing reconstruction, and compare to the systematic contamination of the B-mode power spectrum detection. We consider two instrumental configurations, one with noise sensitivity for polarization of $9.6 \mu \mathrm{K}$-arcmin and FWHM of 8.0' (Exp1 from here on), and another CMBPol like instrument with noise sensitivity for polarization of $2.0 \mu \mathrm{K}$-arcmin and FWHM of 4.0' (reference experiment from here on). In Sec. IV and $\mathrm{V}$ we describe our results and conclude with a discussion of the implications for experiments dedicated to measure primordial B-modes or the secondary lensing signal. We leave discussions of the other three lensing potential estimators $(E E, T E, T B)$ to Appendix.

\section{LENSING FORMALISM}

Gravitational lensing deflects the path of CMB photons from the last scattering surface resulting in a remapping of the CMB temperature/polarization pattern on the sky. In this section, we review the basic lensing calculation for both temperature and polarization fields as the starting point of our discussion. We formulate CMB lensing using the flat-sky approximation [22]. The flat-sky approach simplifies the derivation by replacing summations over Wigner symbols of spherical harmonic moments by integrals involving mode coupling angles [7]. More details of CMB lensing can be found in the nice review paper [41].

At a certain position $\hat{\mathbf{n}}$ on the sky, the observed CMB field $\tilde{X}(\hat{\mathbf{n}})$ is lensed from another direction in the primordial CMB sky $X(\hat{\mathbf{n}}+\mathbf{d}(\hat{\mathbf{n}}))$ at $\mathrm{z}=1090$. The remapping process can be described as

$$
\begin{aligned}
\tilde{T}(\hat{\mathbf{n}}) & =T(\hat{\mathbf{n}}+\mathbf{d}(\hat{\mathbf{n}})), \\
{[\tilde{Q} \pm i \tilde{U}](\hat{\mathbf{n}}) } & =[Q \pm i U](\hat{\mathbf{n}}+\mathbf{d}(\hat{\mathbf{n}})),
\end{aligned}
$$

where $\tilde{T}(\hat{\mathbf{n}})(T(\hat{\mathbf{n}}))$ represents the lensed (unlensed) temperature fluctuation field, $\tilde{Q}(\hat{\mathbf{n}})(Q(\hat{\mathbf{n}}))$ and $\tilde{U}(\hat{\mathbf{n}})(U(\hat{\mathbf{n}}))$ are lensed (unlensed) polarization Stokes parameters, and $\mathbf{d}(\hat{\mathbf{n}})$ is the deflection angle which is related to $\phi(\hat{\mathbf{n}})$, the lensing gravitational potential, by $d(\hat{\mathbf{n}})=\nabla \phi(\hat{\mathbf{n}})$. Here and throughout this paper, we use boldface quantities to identify vectors, and $\tilde{X}(X)$ stands for lensed (unlensed) temperature and polarization fields. The lensing potential $\phi(\hat{\mathbf{n}})$ is given by

$$
\phi(\hat{\mathbf{n}})=-2 \int_{0}^{r_{0}} d r \frac{d_{A}\left(r_{0}-r\right)}{d_{A}(r) d_{A}\left(r_{0}\right)} \Phi(r, r \hat{\mathbf{n}}),
$$

where $d_{A}$ is the comoving distance along the line of sight; $r_{0}$ is the comoving distance to the surface of last scattering, and $\Phi$ is gravitational potential. The lensing remapping process conserves the surface brightness distribution of the CMB, thus does not change the one-point statistics.

The observed temperature and polarization fluctuations also include secondary effects, such as Sunyaev-Zel'dovich (SZ) effect [42] and Integrated Sachs Wolfe (ISW) effect [43], which come from the first order density or potential fluctuation and 
thus also correlate with the lensing deflection angle. We denote these physical contamination to lensing reconstruction by $X^{\sec }(\hat{\mathbf{n}})$. We denote the noise component by $X^{n}(\hat{\mathbf{n}})$. The total observed CMB anisotropy therefore includes the lensed primary signal, any secondary effects, and noise, i.e. $X^{t}(\hat{\mathbf{n}})=\tilde{X}(\hat{\mathbf{n}})+X^{\sec }(\hat{\mathbf{n}})+X^{n}(\hat{\mathbf{n}})$. In the next section, we will introduce another contribution to $X^{t}(\hat{\mathbf{n}})$, which comes from instrumental systematics $X^{s y s}(\hat{\mathbf{n}})$. We define the observed CMB field $X^{\text {obs }}(\hat{\mathbf{n}})=X^{t}(\hat{\mathbf{n}})+X^{s y s}(\hat{\mathbf{n}})$. Here we write the secondary contribution as an independent component from the lensed CMB. However, in reality it is hard to separate $X^{s e c}(\hat{\mathbf{n}})$ from $X^{t}(\hat{\mathbf{n}})$ because secondaries are also lensed by gravitational potentials with deflection angles depending on their redshifts. In this paper, we simply drop the contribution effects $X^{\sec }(\hat{\mathbf{n}})$, as this topic is beyond the focus of this paper. We refer the readers to [22] for a treatment of the secondary anisotropy as a physical contamination to the lensing potential reconstruction analysis ${ }^{1}$.

It is convenient to work in Fourier space. If one considers a small enough patch of sky, spherical harmonic modes can be replaced by Fourier modes. Generalization from the flat-sky to the full sky is straightforward. The Fourier transform of the Taylor expended lensed CMB temperature and polarization field is

$$
\begin{aligned}
\tilde{T}(\mathbf{l}) & =\int d \hat{\mathbf{n}} \tilde{T}(\hat{\mathbf{n}}) e^{-i \cdot \mathbf{l} \cdot \hat{\mathbf{n}}}=T(\mathbf{l})-\int \frac{d^{2} \mathbf{I}^{\prime}}{(2 \pi)^{2}} T\left(\mathbf{I}^{\prime}\right) L\left(\mathbf{l}, \mathbf{l}^{\prime}\right) \\
{[\tilde{E} \pm i \tilde{B}](\mathbf{l}) } & =\int d \hat{\mathbf{n}}[\tilde{Q}(\hat{\mathbf{n}}) \pm i \tilde{U}(\hat{\mathbf{n}})] e^{\mp 2 i \varphi_{1}} e^{-i \mathbf{l} \cdot \hat{\mathbf{n}}}=[E(\mathbf{l}) \pm i B(\mathbf{l})]-\int \frac{d^{2} \mathbf{l}^{\prime}}{(2 \pi)^{2}}\left[E\left(\mathbf{I}^{\prime}\right) \pm i B\left(\mathbf{I}^{\prime}\right)\right] L_{p}\left(\mathbf{l}, \mathbf{l}^{\prime}\right) \\
\phi(\mathbf{l}) & =\int d \hat{\mathbf{n}} \phi(\hat{\mathbf{n}}) e^{-i \mathbf{l} \cdot \hat{\mathbf{n}}}
\end{aligned}
$$

where

$$
\begin{aligned}
L\left(\mathbf{l}, \mathbf{l}^{\prime}\right) & \equiv \phi\left(\mathbf{l}-\mathbf{l}^{\prime}\right)\left[\left(\mathbf{l}-\mathbf{l}^{\prime}\right) \cdot \mathbf{l}^{\prime}\right]+\frac{1}{2} \int \frac{d^{2} \mathbf{l}^{\prime \prime}}{(2 \pi)^{2}} \phi\left(\mathbf{l}^{\prime \prime}\right) \times \phi\left(\mathbf{l}-\mathbf{l}^{\prime}-\mathbf{l}^{\prime \prime}\right)\left(\mathbf{l}^{\prime \prime} \cdot \mathbf{l}^{\prime}\right)\left[\left(\mathbf{l}^{\prime \prime}+\mathbf{l}^{\prime}-\mathbf{l}\right) \cdot \mathbf{l}^{\prime}\right]+\ldots, \\
L_{P}\left(\mathbf{l}, \mathbf{l}^{\prime}\right) & \equiv e^{ \pm 2 i\left(\varphi_{\mathbf{I}^{\prime}}-\varphi_{1}\right)} \phi\left(\mathbf{l}-\mathbf{l}^{\prime}\right)\left[\left(\mathbf{l}-\mathbf{l}^{\prime}\right) \cdot \mathbf{l}^{\prime}\right]+\frac{1}{2} \int \frac{d^{2} \mathbf{l}^{\prime \prime}}{(2 \pi)^{2}} e^{ \pm 2 i\left(\varphi_{\mathbf{I}^{\prime}}-\varphi_{1}\right)} \phi\left(\mathbf{l}^{\prime \prime}\right) \times \phi\left(\mathbf{l}-\mathbf{l}^{\prime}-\mathbf{l}^{\prime \prime}\right)\left(\mathbf{l}^{\prime \prime} \cdot \mathbf{l}^{\prime}\right)\left[\left(\mathbf{l}^{\prime \prime}+\mathbf{l}^{\prime}-\mathbf{l}\right) \cdot \mathbf{l}^{\prime}\right]+\ldots
\end{aligned}
$$

We can immediately see that lensing induces remapping of CMB fields by lensing potential gradients, hence in Fourier space lensing acts as a convolution which couples different harmonic modes. Fourier moments, power spectrum, bispectrum, trispectrum and so on of the CMB fields and the lensing potential can be defined in the usual manner:

$$
\begin{aligned}
\left\langle X^{i}\left(\mathbf{l}_{1}\right) X^{\prime j}\left(\mathbf{l}_{2}\right)\right\rangle \equiv(2 \pi)^{2} \delta_{\mathrm{D}}\left(\mathbf{l}_{1}+\mathbf{l}_{2}\right) C_{X^{i} X^{\prime j}}^{i j}\left(l_{1}\right), \\
\left\langle X^{i}\left(\mathbf{l}_{1}\right) X^{\prime j}\left(\mathbf{l}_{2}\right) X^{\prime \prime k}\left(\mathbf{l}_{3}\right)\right\rangle_{c} \equiv(2 \pi)^{2} \delta_{\mathrm{D}}\left(\mathbf{l}_{1}+\mathbf{l}_{2}+\mathbf{l}_{3}\right) B_{X X^{\prime} X^{\prime \prime}}^{i j k}\left(\mathbf{l}_{1}, \mathbf{l}_{2}, \mathbf{l}_{3}\right), \\
\left\langle X^{i}\left(\mathbf{l}_{1}\right) X^{\prime j}\left(\mathbf{l}_{2}\right) X^{\prime \prime k}\left(\mathbf{l}_{3}\right) X^{\prime \prime \prime m}\left(\mathbf{l}_{4}\right)\right\rangle_{c} \equiv(2 \pi)^{2} \delta_{\mathrm{D}}\left(\mathbf{l}_{1}+\mathbf{l}_{2}+\mathbf{l}_{3}+\mathbf{l}_{4}\right) T_{X X^{\prime} X^{\prime \prime} X^{\prime \prime \prime}}^{i j k m}\left(\mathbf{l}_{1}, \mathbf{l}_{2}, \mathbf{l}_{3}, \mathbf{l}_{4}\right), \\
\ldots
\end{aligned}
$$

where the angle brackets represent ensemble averages over realizations of the primordial CMB fields, the large-scale structure between observers and the last scattering surface, and the experimental noise. The connected part of the $\mathrm{n}$-point function is denoted by the subscript $c$. The fields $X, X^{\prime}, X^{\prime \prime}, X^{\prime \prime \prime}$ are among $\{T(\mathbf{l}), E(\mathbf{l}), B(\mathbf{l}), \phi(\mathbf{l})\}$. The superscripts $i, j, k, m$ represent the unlensed field $X$, the lensed field $\tilde{X}$, the instrumental noise $X^{n}$, the instrumental systematics $X^{\text {sys }}, \mathrm{CMB}$ secondary contribution $X^{s e c}$, the total signal $X^{t}$, or the observed field $X^{o b s}$. We note that the bispectrum and higher order odd-correlations vanish if one ignores the secondary effects. This is because odd moments contain sample averages over the odd primordial CMB fields which we assume to be Gaussian.

We make the assumption that fluctuations in the large-scale structure between the observer and the last scattering surface are Gaussian and hence can be fully described by a power spectrum. We use the lensing potential power spectrum calculated from CAMBFAST. The instrumental noise $X^{n}$ is also assumed to be Gaussian. We note that primordial non-Gaussianity can possibly contribute percent level uncertainty to our analysis which is considered in [45].

We are now in the position to calculate any order (cross) correlation functions of CMB fields and the lensing potential in Fourier space. We will assume uniform Gaussian noise with the power spectrum $C_{l}^{X X \mathrm{n}}$ given by

$$
C_{l}^{X X \mathrm{n}}=w_{X}^{-1} e^{l^{2} \sigma_{b}^{2}} .
$$

\footnotetext{
1 As a note, thermal SZ effect can in principal be separated from the primary fluctuations by its spectral dependence. For the kinetic SZ effect, it was claimed that by using a specially designed estimator [44], it is possible to separate it out from real lensing signal. However, some important secondary contributions such as the ISW effect cannot be separated easily and will lead to additional noise contributions due to correlations with the lensing potentials [22].
} 


\begin{tabular}{|c|c|c|}
\hline \hline$X X^{\prime}$ & ${ }^{1} W_{X X^{\prime}}\left(\mathbf{l}_{1}, \mathbf{l}_{2}\right)$ & ${ }^{2} W_{X X^{\prime}}\left(\mathbf{l}_{1}, \mathbf{l}_{2}\right)$ \\
\hline$T T$ & $\left(\mathbf{L} \cdot \mathbf{l}_{1}\right)$ & $\left(\mathbf{L} \cdot \mathbf{l}_{2}\right)$ \\
$T E$ & $\cos 2\left(\varphi_{\mathbf{l}_{1}}-\varphi_{\mathbf{I}_{2}}\right)\left(\mathbf{L} \cdot \mathbf{l}_{1}\right)$ & $\left(\mathbf{L} \cdot \mathbf{l}_{2}\right)$ \\
$T B$ & $\sin 2\left(\varphi_{\mathbf{l}_{1}}-\varphi_{\mathbf{I}_{2}}\right)\left(\mathbf{L} \cdot \mathbf{l}_{1}\right)$ & 0 \\
$E E$ & $\cos 2\left(\varphi_{\mathbf{l}_{1}}-\varphi_{\mathbf{l}_{2}}\right)\left(\mathbf{L} \cdot \mathbf{l}_{1}\right)$ & $\cos 2\left(\varphi_{\mathbf{l}_{1}}-\varphi_{\mathbf{l}_{2}}\right)\left(\mathbf{L} \cdot \mathbf{l}_{2}\right)$ \\
$E B$ & $\sin 2\left(\varphi_{\mathbf{l}_{1}}-\varphi_{\mathbf{I}_{2}}\right)\left(\mathbf{L} \cdot \mathbf{l}_{1}\right)$ & $\sin 2\left(\varphi_{\mathbf{l}_{1}}-\varphi_{\mathbf{I}_{2}}\right)\left(\mathbf{L} \cdot \mathbf{l}_{2}\right)$ \\
$B B$ & $\cos 2\left(\varphi_{\mathbf{l}_{1}}-\varphi_{\mathbf{l}_{2}}\right)\left(\mathbf{L} \cdot \mathbf{l}_{1}\right)$ & $\cos 2\left(\varphi_{\mathbf{l}_{1}}-\varphi_{\mathbf{l}_{2}}\right)\left(\mathbf{L} \cdot \mathbf{l}_{2}\right)$ \\
\hline
\end{tabular}

TABLE I: Window functions which appear in Eq. 11] and Eq. 12]; here $\mathbf{L}=\mathbf{l}_{1}+\mathbf{l}_{2}$

$w_{X}^{-1}$ is the detector noise variance per steradian area for temperature $(\mathrm{X}=\mathrm{T})$ or polarization $(\mathrm{X}=\mathrm{E}$ or $\mathrm{B})$, and $\sigma_{b}=\theta_{f w h m} / \sqrt{8 \ln 2}$ is the effective beamwidth of the instrument calculated from its full-width half-maximum resolution $\theta_{f w h m}$. We will assume fully polarized detector, for which $2 w_{T}=w_{E}=w_{B}$.

Quadratic combinations of CMB fields can be used as estimators of the lensing potential field and hence the intervening projected mass between us and the last scattering surface. Furthermore, a CMB-field-squared map appropriately filtered in Fourier space can serve as an optimal estimator for the deflection field. Optimal filters for quadratic estimators have been designed [15]

$$
d_{X X^{\prime}}(\mathbf{L}) \equiv \frac{A_{X X^{\prime}}(L)}{L} \int \frac{d^{2} \mathbf{l}_{1}}{(2 \pi)^{2}} X^{\mathrm{t}}\left(\mathbf{l}_{1}\right) X^{\prime \mathrm{t}}\left(\mathbf{l}_{2}\right) F_{X X^{\prime}}\left(\mathbf{l}_{1}, \mathbf{l}_{2}\right),
$$

where $X$ and $X^{\prime}$ can be T, E, and B. The normalization $A_{X X^{\prime}}$ is chosen such that $\left\langle d_{X X^{\prime}}(\mathbf{L})\right\rangle_{\mathrm{CMB}}=d(\mathbf{L}) \equiv L \phi$

$$
A_{X X^{\prime}}(L) \equiv L^{2}\left[\int \frac{d^{2} \mathbf{l}_{1}}{(2 \pi)^{2}} f_{X X^{\prime}}\left(\mathbf{l}_{1}, \mathbf{l}_{2}\right) F_{X X^{\prime}}\left(\mathbf{l}_{1}, \mathbf{l}_{2}\right)\right]^{-1},
$$

where

$$
F_{X X^{\prime}}\left(\mathbf{l}_{1}, \mathbf{l}_{2}\right)=\frac{C_{l_{1}}^{X^{\prime} X^{\prime} \mathrm{t}} C_{l_{2}}^{X X \mathrm{t}} f_{X X^{\prime}}\left(\mathbf{l}_{1}, \mathbf{l}_{2}\right)-C_{l_{1}}^{X X^{\prime} \mathrm{t}} C_{l_{2}}^{X X^{\prime} \mathrm{t}} f_{X X^{\prime}}\left(\mathbf{l}_{2}, \mathbf{l}_{1}\right)}{C_{l_{1}}^{X X \mathrm{t}} C_{l_{2}}^{X^{\prime} X^{\prime} \mathrm{t}} C_{l_{1}}^{X^{\prime} X^{\prime} \mathrm{t}} C_{l_{2}}^{X X \mathrm{t}}-\left(C_{l_{1}}^{X X^{\prime} \mathrm{t}} C_{l_{2}}^{X X^{\prime} \mathrm{t}}\right)^{2}}
$$

where for $X X^{\prime}=T T, E E, B B$, and $T E$,

$$
f_{X X^{\prime}}\left(\mathbf{l}_{1}, \mathbf{l}_{2}\right)=C_{l_{1}}^{X X^{\prime} 1} W_{X X^{\prime}}\left(\mathbf{l}_{1}, \mathbf{l}_{2}\right)+C_{l_{2}}^{X X^{\prime} 2} W_{X X^{\prime}}\left(\mathbf{l}_{1}, \mathbf{l}_{2}\right),
$$

and for $X=\{T, E\}, X^{\prime}=B$,

$$
f_{X X^{\prime}}\left(\mathbf{l}_{1}, \mathbf{l}_{2}\right)=C_{l_{1}}^{X E 1} W_{X X^{\prime}}\left(\mathbf{l}_{1}, \mathbf{l}_{2}\right)+C_{l_{2}}^{X^{\prime} X^{\prime} 2} W_{X X^{\prime}}\left(\mathbf{l}_{1}, \mathbf{l}_{2}\right) .
$$

The window functions $W_{X X^{\prime}}$ are given in Table I In Fig. 1] we show the input power spectrum of the lensed CMB fields, the reconstructed deflection field, and the corresponding Gaussian noise as obtained by using the estimators defined in Eq. (8). Note that the average \langle\rangle$_{\mathrm{CMB}}$ denotes an ensemble average restricted only to different Gaussian realizations of the primordial $\mathrm{CMB}$ and instrument noise but assuming a fixed realization of the large-scale structure. The unmarked average, \langle\rangle , means the average over the primordial CMB field and the large-scale structure realizations as defined in Eq. (6). For the purposes of estimating the large-scale structure in the real observable universe, it is essential to ensure that the estimators after appropriate averaging over realizations are truly unbiased for a typical realization of the primordial Gaussian CMB field. As we will see in the next section that in the presence of non-zero systematic contamination, the estimators are biased.

\section{INSTRUMENTAL SYSTEMATICS EFFECT ON THE LENSING POTENTIAL POWER SPECTRUM}

In this section, we use the quadratic estimators to derive the effects of instrumental systematics on the reconstructed lensing potential power spectrum. We show that instrumental systematics can introduce non-Gaussian correlations of CMB fields. The instrumental systematics-induced CMB trispectrum gives an extra contamination to the reconstructed deflection angle power spectrum. In subsection $\amalg$ w we consider the simple case of the TT estimator to explain how systematics contaminate the lensing reconstruction process. In subsection $\amalg$ ] we take the EB estimator as an example to show how instrumental systematics in CMB polarization measurements affect the lensing reconstruction. In order to compare the experimental requirements for primordial B-mode detection and lensing potential reconstruction, we also calculate the effects of instrumental systematics on B-mode detection for a given inflationary energy scale. 

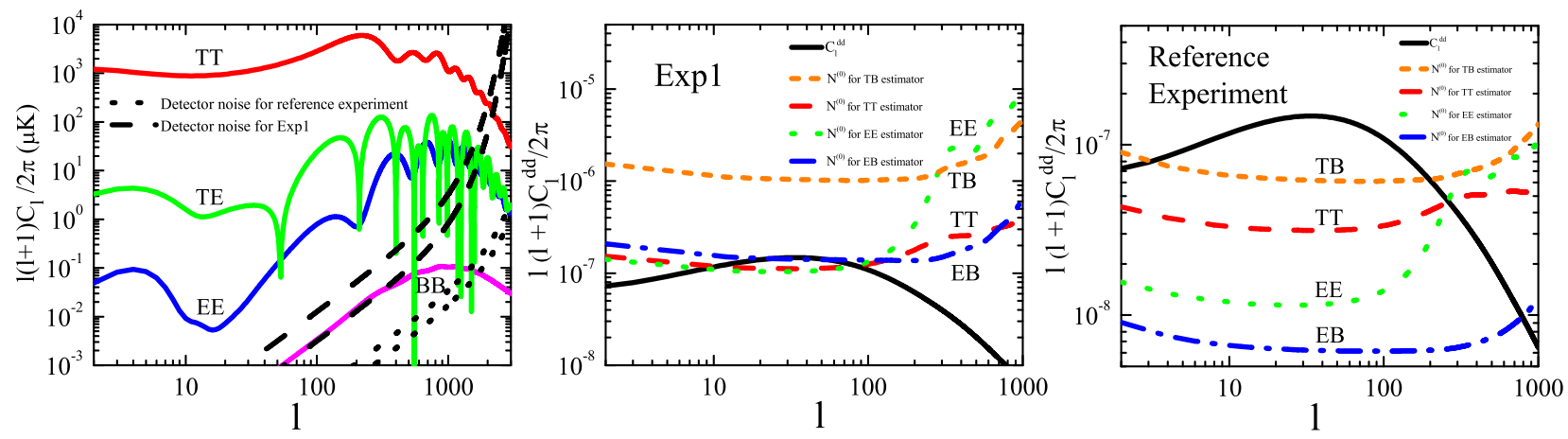

FIG. 1: Left panel: CMB power spectrum for the TT, TE, EE and lensed BB power spectrum. The lower (upper) dashed black line shows the temperature (polarization) noise for Exp1. The lower (upper) dotted black line shows the temperature (polarization) noise for CMBPol-like reference experiment. Center panel: Gaussian noise for different quadratic estimators for the Exp1. Right panel: Same as central panel but for CMBPol-like reference experiment. Note that the EB estimator has the lowest Gaussian noise, and may thus be considered as the best estimator among all the possible quadratic estimators.

\section{A. A simple example of temperature systematics}

We introduce the calibration parameter (gain fluctuation of receivers) $a(\hat{\mathbf{n}})$ for temperature measurement, defined as:

$$
\tilde{T}^{o b s}(\hat{\mathbf{n}})=[1+a(\hat{\mathbf{n}})] \tilde{T}^{t}(\hat{\mathbf{n}}) .
$$

If we assume that there is no correlation between the lensing potential $\phi$ and the instrumental systematic $a(\hat{\mathbf{n}})$, the power spectrum of the lensed CMB temperature with systematics correction term can be found as (more details are given in Appendix $\mathrm{A}$ ):

$$
\tilde{C}_{l}^{T T}=\left[1-\int \frac{d^{2} \mathbf{l}_{1}}{(2 \pi)^{2}} C_{l_{1}}^{\phi \phi}\left(\mathbf{l}_{1} \cdot \mathbf{l}\right)^{2}\right] C_{l}^{T T}+\int \frac{d^{2} \mathbf{l}_{1}}{(2 \pi)^{2}} C_{\left|\mathbf{l}-\mathbf{l}_{\mid}\right|}^{T T} C_{l_{1}}^{\phi \phi}\left[\left(\mathbf{l}-\mathbf{l}_{1}\right) \cdot \mathbf{l}_{1}\right]^{2}+\int \frac{d^{2} \mathbf{l}_{1}}{(2 \pi)^{2}} C_{||-\mathbf{l}_{1} \mid}^{a a} C_{l_{1}}^{T T}
$$

This result is given to linear order in the lensing-potential power spectrum $C_{l}^{\phi \phi}$ and the gain fluctuation systematics power spectrum $C_{I}^{a a}$. The last term represents the bias introduced by the calibration systematics. In the absence of systematic effects, it is easy to prove that the deflection angle estimator is $\left\langle d_{T T}(\mathbf{L})\right\rangle_{\mathrm{CMB}}=L \phi(\mathbf{L}) \equiv d_{T T}(\mathbf{L})$, as desired. But once the contribution from the calibration parameter $a(\hat{\mathbf{n}})$ is considered, one finds:

$$
\left\langle d_{T T}(\mathbf{L})\right\rangle_{\mathrm{CMB}}=d_{T T}(\mathbf{L})+\frac{A_{T T}(L)}{L} \int \frac{d^{2} \mathbf{l}_{1}}{(2 \pi)^{2}} F_{T T}\left(\mathbf{l}_{1}, \mathbf{l}_{2}\right) a(\mathbf{L})\left(C_{l_{1}}^{T T}+C_{l_{2}}^{T T}\right),
$$

i.e. in the presence of $a(\mathbf{L})$ the estimator $d_{T T}(\mathbf{L})$ is a biased estimator for the deflection field in Fourier space. Consequently, the deflection angle power spectrum $\left\langle d_{T T}(\mathbf{L}) d_{T T}(\mathbf{L})\right\rangle$ would be biased due to systematic contamination, and is given by (see Appendix $\mathrm{A}$ for details) 


$$
\begin{aligned}
\left\langle\left\langle\left\langle d_{T T}(\mathbf{L}) \cdot d_{T T}\left(\mathbf{L}^{\prime}\right)\right\rangle_{\mathrm{CMB}}\right\rangle_{\mathrm{LSS}}\right\rangle_{\mathrm{SYS}}= & \frac{A_{T T}(L)}{L} \frac{A_{T T}\left(L^{\prime}\right)}{L^{\prime}} \\
& \times \int \frac{d^{2} \mathbf{l}_{1}}{(2 \pi)^{2}} \int \frac{d^{2} \mathbf{l}_{1}^{\prime}}{(2 \pi)^{2}}(2 \pi)^{2} F_{T T}\left(\mathbf{l}_{1}, \mathbf{l}_{2}\right) F_{T T}\left(\mathbf{l}_{1}{ }^{\prime}, \mathbf{l}_{2}{ }^{\prime}\right) \\
& \left\{C_{L}^{\phi \phi} f_{T T}\left(\mathbf{l}_{1}, \mathbf{l}_{2}\right) f_{T T}\left(\mathbf{l}_{1}{ }^{\prime}, \mathbf{l}_{2}{ }^{\prime}\right) \delta_{\mathrm{D}}\left(\mathbf{L}+\mathbf{L}^{\prime}\right)\right. \\
& +(2 \pi)^{2} C_{l_{1}}^{T T \mathrm{t}} C_{l_{2}}^{T T \mathrm{t}}\left[\delta_{\mathrm{D}}\left(\mathbf{l}_{1}{ }^{\prime}+\mathbf{l}_{1}\right) \delta_{\mathrm{D}}\left(\mathbf{l}_{2}{ }^{\prime}+\mathbf{l}_{2}\right)+\delta_{\mathrm{D}}\left(\mathbf{l}_{2}{ }^{\prime}+\mathbf{l}_{1}\right) \delta_{\mathrm{D}}\left(\mathbf{l}_{1}{ }^{\prime}+\mathbf{l}_{2}\right)\right] \\
& +\left[C_{\mathbf{l}_{1}+\mathbf{l}_{1}{ }^{\prime} \mid}^{\phi} f_{T T}\left(\mathbf{l}_{1}, \mathbf{l}_{1}{ }^{\prime}\right) f_{T T}\left(\mathbf{l}_{2}, \mathbf{l}_{2}{ }^{\prime}\right)+C_{\left|\mathbf{l}_{1}+\mathbf{l}_{2}{ }^{\prime}\right|} f_{T T}\left(\mathbf{l}_{1}, \mathbf{l}_{2}{ }^{\prime}\right) f_{T T}\left(\mathbf{l}_{2}, \mathbf{l}_{1}{ }^{\prime}\right)\right] \delta_{\mathrm{D}}\left(\mathbf{L}+\mathbf{L}^{\prime}\right) \\
& +\left[C_{L}^{a a} f_{a a}\left(\mathbf{l}_{1}, \mathbf{l}_{2}\right) f_{a a}\left(\mathbf{l}_{1}{ }^{\prime}, \mathbf{l}_{2}{ }^{\prime}\right)+C_{\left|\mathbf{l}_{1}+\mathbf{l}_{1}{ }^{\prime}\right|} f_{a a}\left(\mathbf{l}_{1}, \mathbf{l}_{1}{ }^{\prime}\right) f_{a a}\left(\mathbf{l}_{2}, \mathbf{l}_{2}{ }^{\prime}\right)\right. \\
& \left.\left.+C_{\left|\mathbf{l}_{1}+\mathbf{l}_{2}{ }^{\prime}\right|}^{a a} f_{a a}\left(\mathbf{l}_{1}, \mathbf{l}_{2}{ }^{\prime}\right) f_{a a}\left(\mathbf{l}_{2}, \mathbf{l}_{1}{ }^{\prime}\right)\right] \delta_{\mathrm{D}}\left(\mathbf{L}+\mathbf{L}^{\prime}\right)\right\} \\
=(2 & \pi)^{2} \delta_{\mathrm{D}}\left(\mathbf{L}+\mathbf{L}^{\prime}\right)\left[C_{L}^{d d}+N_{T T, T T}^{(0)}(L)+N_{T T, T T}^{(1)}(L)+N_{T T, T T}^{(S)}(L)+\ldots\right],
\end{aligned}
$$

where we define $f_{a a}\left(\mathbf{l}_{1}, \mathbf{l}_{2}\right)=C_{l_{1}}^{T T}+C_{l_{2}}^{T T}$. In the last line, the first term in the square bracket is the deflection angle power spectrum $C_{L}^{d d}$. The second term is the so called Gaussian noise $N_{T T, T T}^{(0)}(L)$ which gives the dominant noise contribution to the variance of the deflection power spectrum. The third term $N_{T T, T T}^{(1)}(L)$ is the the leading order non-Gaussian noise which is first order in $C_{L}^{\phi \phi}$ and gives correction to the dominant Gaussian noise $N_{T T, T T}^{(0)}(L)$. The forth term, $N_{T T, T T}^{(S)}(L)$, is the leading order instrumental systematic contribution to the variance and is first order in $C_{L}^{S S}$. The Gaussian noise $N_{T T, T T}^{(0)}(L)$ and the first order non-Gaussian noise $N_{T T, T T}^{(1)}(L)$ have been previously calculated in [15] and [21], respectively. The systematic noise term $N_{T T, T T}^{(S)}(L)$ is a new contribution to lensing power spectrum. In principle, one should include noise terms which are higher order in $C_{L}^{\phi \phi}$ and $C_{L}^{S S}$, however since both of them are small, we truncate at the first order and expect that higher order contributions are much smaller.

The quadratic estimator given in Eq. (8) is optimized in the presence of $N_{T T, T T}^{(0)}(L)$, and assuming no contribution from the first order non-Gaussian noise $N_{T T, T T}^{(1)}(L)$ and instrumental systematic noise $N_{T T, T T}^{(S)}(L)$. Hence the estimator is optimal ${ }^{2}$ as long as $N_{T T, T T}^{(1)}(L) \ll N_{T T, T T}^{(0)}(L)$, and $N_{T T, T T}^{(S)}(L) \ll N_{T T, T T}^{(0)}(L)$. It has been shown that the non-Gaussian noise $N_{T T, T T}^{(1)}(L)$ is about one order of magnitude smaller than the Gaussian noise contribution [21]. In $\amalg$ ] we calculate the systematic noise term $N^{S}(L)$ contribution to the lensing potential reconstruction for the EB estimator.

\section{B. General analysis on polarization systematics}

We parametrize the fields of instrumental systematics for CMB polarization measurements following [32]. The polarization contaminations fall into two categories, one associated with the detector system which distorts the polarization state of the incoming polarized signal (Type I hereafter), and another associated with distortion of the CMB signal due to the beam anisotropy (Type II hereafter). This parametrization can be generalized to different polarimeters. The instrumental response to incoming CMB radiation is usually described by the Jones transfer matrix. Bias induced in the matrix determination will mix the Stokes parameters determined from it. To first order, the effect of Type I systematics on the Stokes parameters can be written as [32]

$$
\delta[Q \pm i U](\hat{\mathbf{n}})=[a \pm i 2 \omega](\hat{\mathbf{n}})[Q \pm i U](\hat{\mathbf{n}})+\left[f_{1} \pm i f_{2}\right](\hat{\mathbf{n}})[Q \mp i U](\hat{\mathbf{n}})+\left[\gamma_{1} \pm i \gamma_{2}\right](\hat{\mathbf{n}}) T(\hat{\mathbf{n}}) .
$$

$a$ is a scalar field which describes the miscalibration of the polarization measurements (recall that in last subsection, we used $a$ to denote the miscalibration of temperature measurements), $\omega$ is also a scalar field that describes the rotation angle of the instrument, $\left(f_{1} \pm i f_{2}\right)$ are spin \pm 4 fields that describe the coupling between two spin states (spin-flip), and $\left(\gamma_{1} \pm i \gamma_{2}\right)$ are spin \pm 2 fields that describe monopole leakage from the temperature to polarization.

\footnotetext{
${ }^{2}$ If the systematic contributions are comparable to the Gaussian noise contribution then we need to design new optimal lensing reconstruction estimators to take into account the instrumental systematics effect.
} 
Similar to the Type I systematics, the effect of Type II systematics on the Stokes parameters can be written as [32]

$$
\delta[Q \pm i U](\hat{\mathbf{n}} ; \sigma)=\sigma \mathbf{p}(\hat{\mathbf{n}}) \cdot \nabla[Q \pm i U](\hat{\mathbf{n}} ; \sigma)+\sigma\left[d_{1} \pm i d_{2}\right](\hat{\mathbf{n}})\left[\partial_{1} \pm i \partial_{2}\right] T(\hat{\mathbf{n}} ; \sigma)+\sigma^{2} q(\hat{\mathbf{n}})\left[\partial_{1} \pm i \partial_{2}\right]^{2} T(\hat{\mathbf{n}} ; \sigma)
$$

the systematic fields are smoothed over the average beam $\sigma$ of the experiment. Therefore the type II systematic fields are sensitive to the imperfection of the beam on the scale $\sigma .\left(p_{1} \pm i p_{2}\right)$ are spin \pm 1 fields that describe pointing errors, $\left(d_{1} \pm i d_{2}\right)$ are also spin \pm 1 fields that describe dipole leakage from temperature to polarization, and $q$ is a scalar field that describes quadrupole leakage [32].

As a simple model, we will assume that the contamination fields, as defined in (17) and (18), are statistically isotropic and Gaussian (although some of the systematics fields need not be so), thus their statistical properties can be fully described by their power spectra,

$$
\left\langle S(\mathbf{l}) S\left(\mathbf{l}^{\prime}\right)\right\rangle=(2 \pi)^{2} \delta\left(\mathbf{l}+\mathbf{l}^{\prime}\right) C_{l}^{S S}
$$

where $S$ stands for any of the 11 systematic fields. The systematic fields can be modeled with the power spectra of the form

$$
C_{l}^{S S}=C_{0} \exp \left(-l(l+1) \alpha_{S}^{2}\right)
$$

i.e. white noise above certain coherence scale $\alpha_{S}$, which is a key quantity to affect the level of contamination of each systematics effects. The normalization factor $C_{0}$ can be determined by

$$
C_{0}^{2}=A_{S}^{2}\left[\int \frac{d^{2} l}{(2 \pi)^{2}} \exp \left(-l(l+1) \alpha_{S}^{2}\right)\right]^{-1},
$$

where $A_{S}$ characterizes the rms of the contamination field $S$.

The instrumental systematics induce distortions on the CMB fileds. The contaminations to the $B B$ and $E E$ power spectra due to different measurement systematics take the form

$$
\begin{aligned}
& \delta C_{l}^{B B}=\sum_{S S^{\prime}} \int \frac{d^{2} \mathbf{l}^{\prime}}{(2 \pi)^{2}} C_{\left|\mathbf{l}-\mathbf{I}^{\prime}\right|}^{S S^{\prime}} C_{\left|\mathbf{I}^{\prime}\right|}^{E E}(\sigma)\left[W_{B}^{S}\left(\mathbf{l}, \mathbf{l}^{\prime}\right)\right]^{2}+\sum_{S S^{\prime}} \int \frac{d^{2} \mathbf{l}^{\prime}}{(2 \pi)^{2}} C_{\left|\mathbf{l}-\mathbf{I}^{\prime}\right|}^{S S^{\prime}} C_{\left|\mathbf{I}^{\prime}\right|}^{T T}(\sigma)\left[W_{B}^{S}\left(\mathbf{l}, \mathbf{l}^{\prime}\right)\right]^{2}, \\
& \delta C_{l}^{E E}=\sum_{S S^{\prime}} \int \frac{d^{2} \mathbf{l}^{\prime}}{(2 \pi)^{2}} C_{\left|\mathbf{l}-\mathbf{l}^{\prime}\right|}^{S S^{\prime} \mid} C_{\left|\mathbf{I}^{\prime}\right|}^{E E}(\sigma)\left[W_{E}^{S}\left(\mathbf{l}, \mathbf{l}^{\prime}\right)\right]^{2}+\sum_{S S^{\prime}} \int \frac{d^{2} \mathbf{l}^{\prime}}{(2 \pi)^{2}} C_{\left|\mathbf{l}-\mathbf{I}^{\prime}\right|}^{S S^{\prime}} C_{\left|\mathbf{I}^{\prime}\right|}^{T T}(\sigma)\left[W_{E}^{S}\left(\mathbf{l}, \mathbf{l}^{\prime}\right)\right]^{2} .
\end{aligned}
$$

The explicit forms of $W_{B}^{S}\left(\mathbf{l}_{1}, \mathbf{l}_{2}\right)$ and $W_{E}^{S}\left(\mathbf{l}_{1}, \mathbf{l}_{2}\right)$ are given in Table $\Pi$ which are the window functions of each systematic $S$ for B-mode and E-mode harmonics, respectively. The summations of the first term on the RHS of Eq. (22) and Eq. 23) run over calibration $a$, rotation $\omega$, spin flip $f_{a}$ and $f_{b}$, and pointing error $\gamma_{a}$ and $\gamma_{b}$. The summations of the second term run over the rest of the systematics parameters which describe the temperature leakage given in Table $C_{l}^{E E}(\sigma)$ and $C_{l}^{T T}(\sigma)$ are the beam smoothed temperature and E-mode polarization power spectra.

$$
C_{l}^{E E}(\sigma)=C_{l}^{E E} \exp (-l(l+1) \sigma), \quad C_{l}^{T T}(\sigma)=C_{l}^{T T} \exp (-l(l+1) \sigma) .
$$

We use Eq. (22) and Eq. (23) to calculate the systematic requirements for B-mode detection in order to compare the requirements for lensing reconstruction. We show the results in Tables III and IV

Now we move on to calculate the systematic contamination on the lensing power spectrum. The polarization fields are essentially uncorrelated with the lensing potential, so if we do not consider secondary effects, the $n$-point functions with $n$ odd are zero. The next non-zero order is the trispectrum. The calculation for the connected part of the trispectrum of polarization in the presence of instrumental systematics is similar to the connected temperature trispectrum presented in the last section. Here we give the results for the trispectrum related to lensing reconstruction using EB estimator, and refer the readers to the Appendix $C$ for the explicit calculation for other quadratic estimators. At leading order we have 


\begin{tabular}{||c||c|c||}
\hline \hline Type of $\mathbf{S}$ & $W_{B}^{S}\left(\mathbf{l}_{1}, \mathbf{l}_{2}\right)$ & $W_{E}^{S}\left(\mathbf{l}_{1}, \mathbf{l}_{2}\right)$ \\
\hline Calibration $a$ & $\sin \left[2\left(\varphi_{l_{2}}-\phi_{L}\right)\right]$ & $\cos \left[2\left(\varphi_{l_{2}}-\varphi_{L}\right)\right]$ \\
Rotation $\omega$ & $2 \cos \left[2\left(\varphi_{l_{2}}-\varphi_{L}\right)\right]$ & $-2 \sin \left[2\left(\varphi_{l_{2}}-\varphi_{L}\right)\right]$ \\
Pointing $p_{a}$ & $\sigma\left(\mathbf{l}_{2} \times \hat{\mathbf{l}}_{1}\right) \cdot \hat{\mathbf{z}} \sin \left[2\left(\varphi_{l_{2}}-\varphi_{L}\right)\right]$ & $\sigma\left(\mathbf{l}_{2} \cdot \hat{\mathbf{l}}_{1}\right) \sin \left[2\left(\varphi_{l_{2}}-\varphi_{l}\right)\right]$ \\
Pointing $p_{b}$ & $\sigma\left(\mathbf{l}_{2} \cdot \hat{\mathbf{l}}_{1}\right) \sin \left[2\left(\varphi_{l_{2}}-\varphi_{l}\right)\right]$ & $-\sigma\left(\mathbf{l}_{2} \times \hat{\mathbf{l}}_{1}\right) \cdot \hat{\mathbf{z}} \sin \left[2\left(\varphi_{l_{2}}-\varphi_{L}\right)\right]$ \\
Flip $f_{a}$ & $\sin \left[2\left(2 \varphi_{l_{1}}-\varphi_{l_{2}}-\varphi_{L}\right)\right]$ & $\cos \left[2\left(2 \varphi_{l_{1}}-\varphi_{l_{2}}-\varphi_{L}\right)\right]$ \\
Flip $f_{b}$ & $\cos \left[2\left(2 \varphi_{l_{1}}-\varphi_{l_{2}}-\varphi_{L}\right)\right]$ & $-\sin \left[2\left(2 \varphi_{l_{1}}-\varphi_{l_{2}}-\varphi_{L}\right)\right]$ \\
Monopole $\gamma_{a}$ & $\sin \left[2\left(\varphi_{l_{1}}-\varphi_{l}\right)\right]$ & $\cos \left[2\left(\phi_{l_{1}}-\varphi_{l}\right)\right]$ \\
Monopole $\gamma_{b}$ & $\cos \left[2\left(\varphi_{l_{1}}-\varphi_{l}\right)\right]$ & $-\sin \left[2\left(\varphi_{l_{1}}-\phi_{l}\right)\right.$ \\
Dipole $d_{a}$ & $-\left(l_{2} \sigma\right) \cos \left[\varphi_{l_{1}}+\varphi_{l_{2}}-2 \varphi_{l}\right]$ & $\left(l_{2} \sigma\right) \sin \left[\varphi_{l_{1}}+\phi_{l_{2}}-2 \varphi_{l}\right]$ \\
Dipole $d_{b}$ & $\left(l_{2} \sigma\right) \sin \left[\varphi_{l_{1}}+\phi_{l_{2}}-2 \varphi_{l}\right]$ & $\left(l_{2} \sigma\right) \cos \left[\varphi_{l_{1}}+\varphi_{l_{2}}-2 \varphi_{l}\right]$ \\
Quadrupole $q$ & $-\left(l_{2} \sigma\right)^{2} \sin \left[2\left(\varphi_{l_{2}}-\varphi_{l}\right)\right]$ & $-\left(l_{2} \sigma\right)^{2} \cos \left[2\left(\varphi_{l_{2}}-\varphi_{l}\right)\right]$ \\
\hline
\end{tabular}

TABLE II: Window functions for all the 11 systematic parameters. First column indicates the type of systematic parameters in consideration. Second and third columns show window functions for systematics induced B-mode $W_{B}^{S}\left(\mathbf{l}_{1}, \mathbf{l}_{2}\right)$, and for E-mode $W_{E}^{S}\left(\mathbf{l}_{1}, \mathbf{l}_{2}\right)$ respectively. These window functions are needed to calculate systematic contamination on primordial gravitational wave detection or the deflection angle power spectrum reconstruction. We note that $\mathbf{l}_{1}=l_{1} \hat{\mathbf{l}}_{1}, \mathbf{l}_{2}=\mathbf{L}-\mathbf{l}_{1}$, and $\mathbf{l}_{2}=l_{2} \hat{\mathbf{l}}_{2}$.

$$
\begin{aligned}
& \left\langle\tilde{E}\left(\mathbf{l}_{1}\right)^{\mathrm{obs}} \tilde{B}\left(\mathbf{l}_{2}\right)^{\mathrm{obs}} \tilde{E}\left(\mathbf{l}_{1}^{\prime}\right)^{\mathrm{obs}} \tilde{B}\left(\mathbf{l}_{2}^{\prime}\right)^{\mathrm{obs}}\right\rangle_{c}=(2 \pi)^{2} \delta_{\mathrm{D}}\left(\mathbf{l}_{1}+\mathbf{l}_{2}+\mathbf{l}_{1}^{\prime}+\mathbf{l}_{2}^{\prime}\right) \times \\
& \left\{C _ { l _ { 1 } } ^ { E E } C _ { l _ { 1 } ^ { \prime } } ^ { E E } \left[C_{\left|\mathbf{l}_{1}+\mathbf{l}_{2}\right|}^{\phi \phi} W_{B}\left(\mathbf{l}_{2},-\mathbf{l}_{1}\right) W_{B}\left(\mathbf{l}_{2}^{\prime},-\mathbf{l}_{1}^{\prime}\right)+C_{\left|\mathbf{l}_{1}+\mathbf{l}_{2}^{\prime}\right|}^{\phi \phi} W_{B}\left(\mathbf{l}_{2},-\mathbf{l}_{1}^{\prime}\right) W_{B}\left(\mathbf{l}_{2}^{\prime},-\mathbf{l}_{1}\right)\right.\right. \\
& \left.+\sum_{S}^{P \text {-distortion }} C_{\left|\mathbf{l}_{1}+\mathbf{l}_{2}\right|}^{S S} W_{B}^{S}\left(\mathbf{l}_{2},-\mathbf{l}_{1}\right) W_{B}^{S}\left(\mathbf{l}_{2}^{\prime},-\mathbf{l}_{1}^{\prime}\right)+\sum_{S}^{P \text {-distortion }} C_{\left|\mathbf{l}_{1}+\mathbf{l}_{2}^{\prime}\right|}^{S S} W_{B}^{S}\left(\mathbf{l}_{2},-\mathbf{l}_{1}^{\prime}\right) W_{B}^{S}\left(\mathbf{l}_{2}^{\prime},-\mathbf{l}_{1}\right)\right] \\
& +C_{l_{1}}^{T E} C_{l_{1}^{\prime}}^{T E}\left[\sum_{S}^{T \text {-leakage }} C_{\left|\mathbf{l}_{1}+\mathbf{l}_{2}\right|}^{S S} W_{B}^{S}\left(\mathbf{l}_{2},-\mathbf{l}_{1}\right) W_{B}^{S}\left(\mathbf{l}_{2}^{\prime},-\mathbf{l}_{1}^{\prime}\right)+\right. \\
& \left.\left.\sum_{S}^{T \text {-leakage }} C_{\mid \mathbf{l}_{1}+\mathbf{l}_{2}^{\prime}}^{S S} W_{B}^{S}\left(\mathbf{l}_{2},-\mathbf{l}_{1}^{\prime}\right) W_{B}^{S}\left(\mathbf{l}_{2}^{\prime},-\mathbf{l}_{1}\right)\right]\right\}
\end{aligned}
$$

where we defined the lensing B-mode window function $W_{B}\left(\mathbf{l}, \mathbf{l}^{\prime}\right) \equiv \mathbf{l}^{\prime} \cdot\left(\mathbf{l}-\mathbf{l}^{\prime}\right) \sin 2\left(\varphi_{\mathbf{l}}-\varphi_{\mathbf{I}^{\prime}}\right)$ and $W_{B}^{S}\left(\mathbf{l}_{1},-\mathbf{l}_{1}^{\prime}\right)$ is the systematics window function for any of the 11 systematics parameters. The formula for each of the systematic window functions $W_{B}^{S}\left(\mathbf{l}, \mathbf{I}^{\prime}\right)$ can be found in Table

Different trispectrum can be constructed from combinations of the temperature and polarization fields. We discuss other cases in the Appendix C in order to calculate EE, TE and TB estimators including contributions from systematics contamination. The formulas shown here are readily generalized to the full sky. For a discussion of the spherical generalization of the polarization trispectra, see [47]. We have shown that E and B-modes are mixed not only by weak lensing, but also by instrumental systematics. Even if there is no lensing induced correlation, certain kinds of systematics can give a non-zero contributions to the trispectrum. Now we move on to construct quadratic lensing estimators from E/B polarization modes, and we quantitatively show how instrumental systematics contamination affects the lensing reconstruction process. Again we take the EB estimator as an example, and leave the discussions of other estimators to the Appendix $\mathrm{C}$ The variance of the deflection angle power spectrum including systematic effects can be written as

$$
\begin{aligned}
\left\langle\left\langle\left\langle d_{E B}(\mathbf{L}) \cdot d_{E B}\left(\mathbf{L}^{\prime}\right)\right\rangle_{\mathrm{CMB}}\right\rangle_{\mathrm{LSS}}\right\rangle_{\mathrm{SYS}}= & \frac{A_{E B}(L)}{L} \frac{A_{E B}\left(L^{\prime}\right)}{L^{\prime}} \int \frac{d^{2} \mathbf{l}_{1}}{(2 \pi)^{2}} \int \frac{d^{2} \mathbf{l}_{1}^{\prime}}{(2 \pi)^{2}} F_{E B}\left(\mathbf{l}_{1}, \mathbf{l}_{2}\right) F_{E B}\left(\mathbf{l}_{1}{ }^{\prime}, \mathbf{l}_{2}{ }^{\prime}\right) \\
& \times\left\langle\tilde{E}\left(\mathbf{l}_{1}\right)^{\mathrm{obs}} \tilde{B}\left(\mathbf{l}_{2}\right)^{\mathrm{obs}} \tilde{E}\left(\mathbf{l}_{1}^{\prime}\right)^{\mathrm{obs}} \tilde{B}\left(\mathbf{l}_{2}^{\prime}\right)^{\mathrm{obs}}\right\rangle \\
= & (2 \pi)^{2} \delta_{\mathrm{D}}\left(\mathbf{L}+\mathbf{L}^{\prime}\right)\left[C^{d d}(L)+N_{E B, E B}^{(0)}(L)+N_{E B, E B}^{(1)}(L)+N_{E B, E B}^{(S)}(L)+\ldots\right],
\end{aligned}
$$

where $\mathbf{L}=\mathbf{I}_{1}+\mathbf{l}_{2}$, and $C^{d d}(L)$ is the deflection angle power spectrum. The terms $N_{E B, E B}^{(0)}(L), N_{E B, E B}^{(1)}(L)$, and $N_{E B, E B}^{(S)}(L)$ are the Gaussian noise, first order non-Gaussian noise, and the first order systematics noise. The Gaussian noise contribution comes 

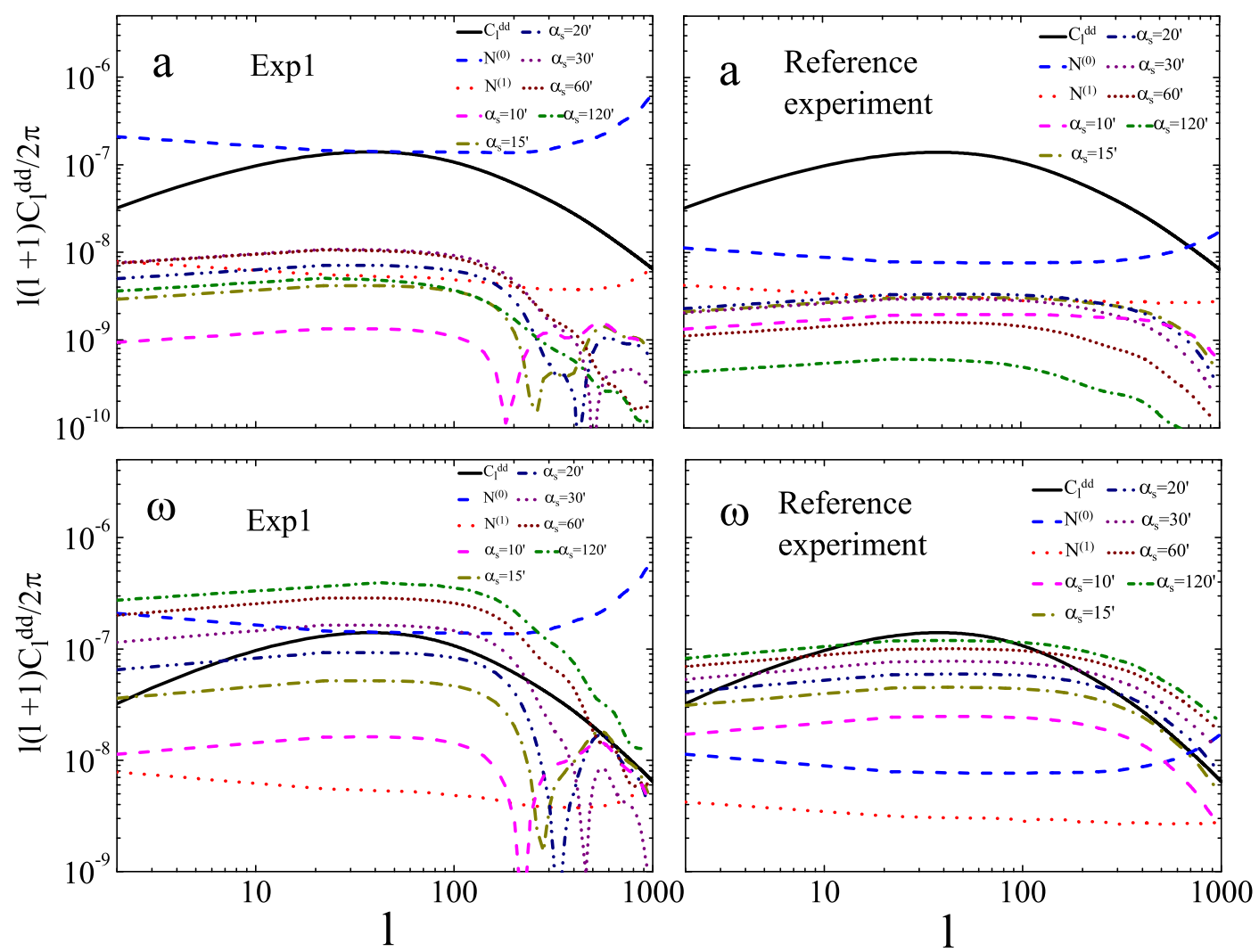

FIG. 2: Upper panels: Contamination from the calibration systematics $a$ to the deflection angle power spectrum using the EB estimator. The rms fluctuation, $A_{S}$ is assumed to be $10 \%$. The left and right panel are for Exp1 and reference experiment respectively. In both the panels, the solid black, dashed blue, and dot red curves show deflection angle power spectrum $C^{d d}(L)$, Gaussian noise $N^{(0)}(L)$, and the first order non-Gaussian noise $N^{(1)}(L)$. The remaining curves show the absolute value of the systematic bias for various choices of coherence length $\alpha_{s}$, starting from $\alpha_{s}=10^{\prime}$ to $\alpha_{s}=120^{\prime}$. Lower panels: Same as the upper panels but for rotation systematics $\omega$.

from the disconnected part of the four-point function, while both the first-order non-Gaussian noise $N_{E B, E B}^{(1)}(L)$ and systematics noise $N_{E B E B}^{(S)}(L)$ contribution comes from the connected part. In deriving Eq. (26) we have used filters $F_{E B}$ which are given in Eq. (10), and the trispectrum which is given in Eq. (25). The ellipses stands for terms beyond first order in the systematics power spectra or the deflection angle power spectrum. We note that the Gaussian noise term also includes systematic effects implicitly since instrumental systematics bias the measured power spectrum as we have shown. The Gaussian and non-Gaussian noise terms in the absence of systematic contribution have been previously reported in [15] and [21]. The systematics noise term $N_{E B, E B}^{(S)}(L)$ is new, for which the explicit form is given as

$$
\begin{aligned}
N_{E B, E B}^{(S)}(L)= & \frac{A_{E B}(L)}{L} \frac{A_{E B}\left(L^{\prime}\right)}{L^{\prime}} \int \frac{d^{2} \mathbf{l}_{1}}{(2 \pi)^{2}} \int \frac{d^{2} \mathbf{l}_{1}^{\prime}}{(2 \pi)^{2}} F_{E B}\left(\mathbf{l}_{1}, \mathbf{l}_{2}\right) F_{E B}\left(\mathbf{l}_{1}{ }^{\prime}, \mathbf{l}_{2}{ }^{\prime}\right) \\
& \left\{C_{l_{1}}^{E E} C_{l_{1}^{\prime}}^{E E}\left[\sum_{S}^{P-\text { distortion }} C_{\left|\mathbf{l}_{1}+\mathbf{l}_{2}\right|}^{S S} W_{B}^{S}\left(\mathbf{l}_{2},-\mathbf{l}_{1}\right) W_{B}^{S}\left(\mathbf{l}_{2}^{\prime},-\mathbf{l}_{1}^{\prime}\right)+\sum_{S}^{P \text {-distortion }} C_{\left|\mathbf{l}_{1}+\mathbf{l}_{2}^{\prime}\right|}^{S S} W_{B}^{S}\left(\mathbf{l}_{2},-\mathbf{l}_{1}^{\prime}\right) W_{B}^{S}\left(\mathbf{l}_{2}^{\prime},-\mathbf{l}_{1}\right)\right]\right. \\
& \left.+C_{l_{1}}^{T E} C_{l_{1}^{\prime}}^{T E}\left[\sum_{S}^{T-\text { leakage }} C_{\left|\mathbf{l}_{1}+\mathbf{l}_{2}\right|}^{S S} W_{B}^{S}\left(\mathbf{l}_{2},-\mathbf{l}_{1}\right) W_{B}^{S}\left(\mathbf{l}_{2}^{\prime},-\mathbf{l}_{1}^{\prime}\right)+\sum_{S}^{T-\text { leakage }} C_{\left|\mathbf{l}_{1}+\mathbf{l}_{2}^{\prime}\right|}^{S S} W_{B}^{S}\left(\mathbf{l}_{2},-\mathbf{l}_{1}^{\prime}\right) W_{B}^{S}\left(\mathbf{l}_{2}^{\prime},-\mathbf{l}_{1}\right)\right]\right\} .
\end{aligned}
$$

Eq. (27) is our main result for the systematics contamination on lensing power reconstruction using the EB estimator. We will use this equation to numerically compute the systematic-induced bias for the 11 systematic parameters. The results are shown 

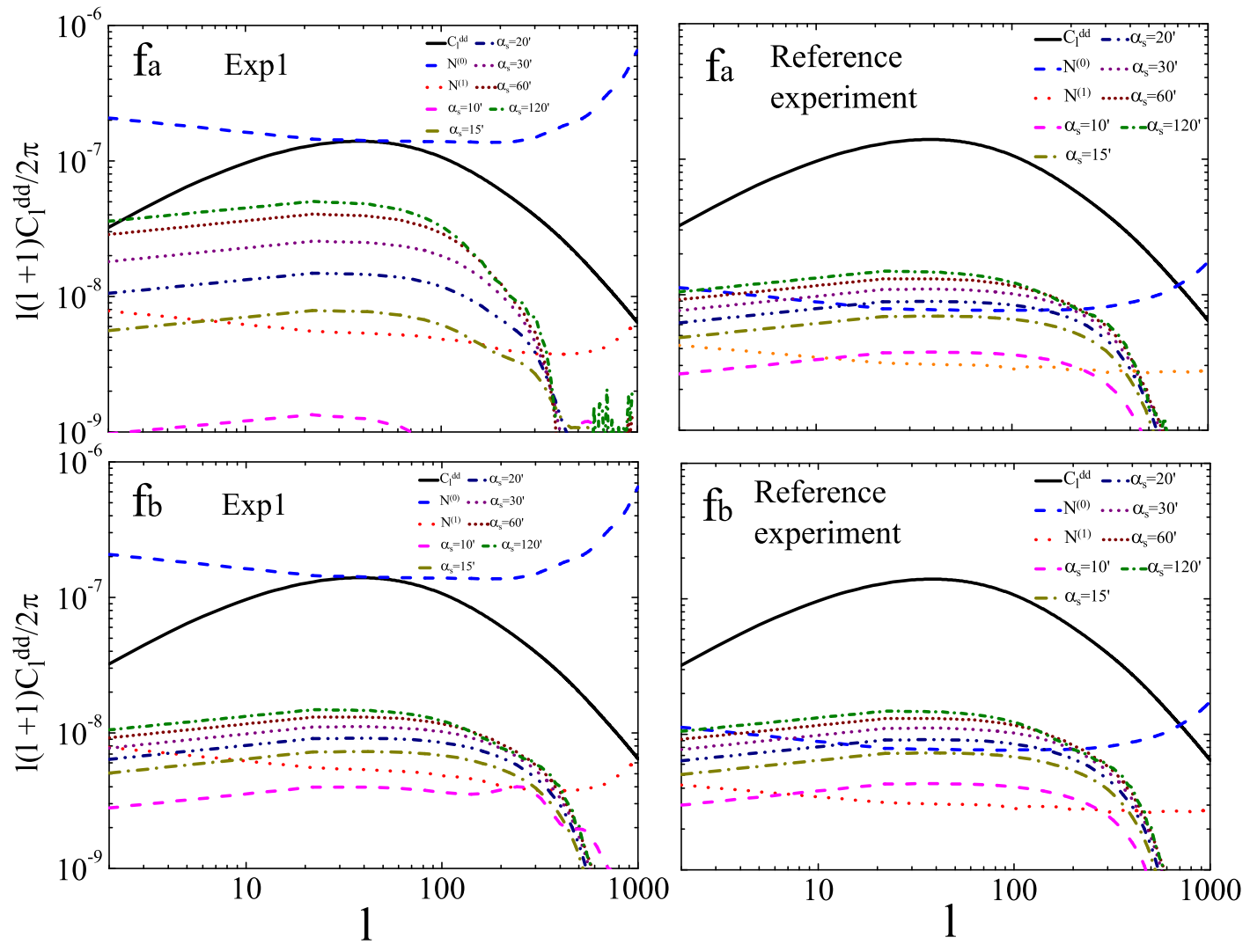

FIG. 3: Upper panels: Contamination from the spin-flip systematics $f_{a}$ to the deflection angle power spectrum using the EB estimator. The rms fluctuation, $A_{S}$ is assumed to be $10 \%$. The left and right panel are for Exp1 and reference experiment respectively. In both the panels, the solid black, dashed blue, and dot red curves show deflection angle power spectrum $C^{d d}(L)$, Gaussian noise $N^{(0)}(L)$, and the first order non-Gaussian noise $N^{(1)}(L)$. The remaining curves show the absolute value of the systematic bias for various choices of coherence length $\alpha_{s}$, starting from $\alpha_{s}=10^{\prime}$ to $\alpha_{s}=120^{\prime}$. Lower panels: Same as the upper panels but for spin-flip systematics $f_{b}$.

in Figures (2) to (7) and are discussed in next section ${ }^{3}$.

\section{RESULTS}

Figures 2 to 7 and Tables [II \& IV summarize our main findings. We have focused on the systematics-induced bias for the $E B$ estimator (results for the other estimators are provided in the Appendix C) because it has the highest Gaussian signal-to-noise ratio for reconstructing the projected matter power spectrum (See Fig. 1] or [15]). Figures 2] 7] show the contamination introduced by different systematic effects (the term $N_{E B, E B}^{(S)}(L)$ in Eq. (27)) in the deflection angle power spectrum reconstruction. We have assumed the rms fluctuation of the systematics fields to be $10 \%$, and varied coherence length starting from minimum $\alpha_{s}=10^{\prime}$ to the maximum $\alpha_{s}=120^{\prime}$. For comparison, we also show the level of Gaussian noise and first order non-Gaussian noise of the EB estimator, the terms $N_{E B, E B}^{(0)}(L)$ and $N_{E B, E B}^{(1)}(L)$ respectively in Eq. (27). Systematics-induced bias generally increases with

\footnotetext{
${ }^{3}$ As a note, for each instrumental systematics parameter $S$, for both the polarization distortion and temperature leakage, there are two terms which contribute to the final results. The terms proportional $C_{\left|\mathbf{I}_{1}+\mathbf{I}_{2}\right|}^{S S}$ is generally much smaller than the terms proportional to $C_{\left|\mathbf{I}_{1}+\mathbf{I}_{2}^{\prime}\right|}^{S S}$ but for non-Gaussian noise $N^{(1)}[21]$, the

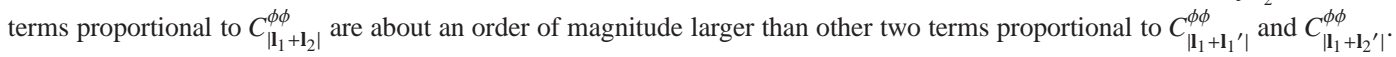




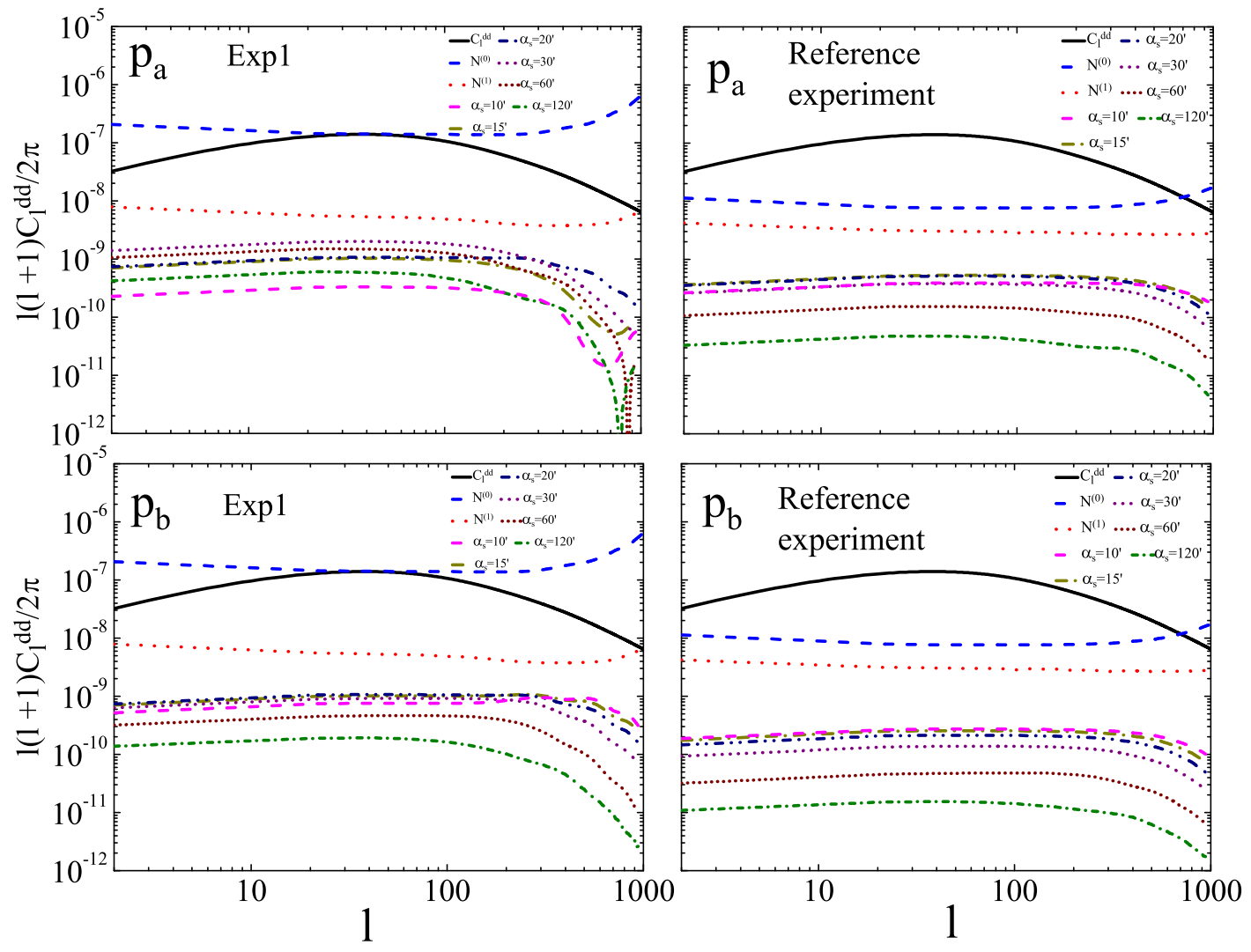

FIG. 4: Upper panels: Contamination from the pointing systematics $p_{a}$ to the deflection angle power spectrum using the EB estimator. The rms fluctuation, $A_{S}$ is assumed to be $10 \%$. The left and right panel are for Exp1 and reference experiment respectively. In both the panels, the solid black, dashed blue, and dot red curves show deflection angle power spectrum $C^{d d}(L)$, Gaussian noise $N^{(0)}(L)$, and the first order non-Gaussian noise $N^{(1)}(L)$. The remaining curves show the absolute value of the systematic bias for various choices of coherence length $\alpha_{s}$, starting from $\alpha_{s}=10^{\prime}$ to $\alpha_{s}=120^{\prime}$. Lower panels: Same as upper panels but for the pointing systematics $p_{b}$.

the increase in coherence length. For all the coherence lengths, the bias $N_{E B, E B}^{(S)}(L)$ is fairly constant on large scales, similar to the feature of the Gaussian noise and the first order non-Gaussian noise of the estimator, however on small scales $(\ell \gtrsim 200)$, the systematic contamination are not constant, and for some systematics, they oscillate between positive and negative values. The fluctuating features are due to the oscillation of Gaussian noise at high $\ell$ and the CMB power spectra included in the systematic calculations, smoothed and filtered by certain window functions. The negative systematic contributions at certain multiple range for some parameters are caused by combining the effects from window functions, which sometimes give negative, and TE cross correlation which enters the calculation of monopole leakage, dipole leakage, and quadrupole leakage from temperature field.

In Table III we calculate the maximum $r m s$ amplitude $A_{s}$ required for each systematic parameters to keep its own contamination lower than the deflection angle power spectrum. Lensing extraction requires the control of systematic rms fluctuations at levels depending on the type of systematics. Among all the 11 systematic parameters, rotation $\omega$, and monopole leakage $\gamma_{a} \& \gamma_{b}$ place the most stringent requirements, while pointing error $p_{a} \& p_{b}$, quadrupole leakage $q$, and calibration $a$ are among the least demanding. Also with respect to sensitivity to the coherence length, some systematic parameters such as rotation $\omega$ and monopole leakage $\gamma_{a} \& \gamma_{b}$ are very sensitive (as shown in Figure (2) and (5), while some are not very sensitive.

For comparison we also show the systematics requirements for primordial B-modes detection. For B-modes we consider two inflationary energy scales $E=1.0 \times 10^{16} \mathrm{GeV}$ and $E=3.0 \times 10^{16} \mathrm{GeV}$. In addition we consider two choices of coherence length $\alpha_{s}=10^{\prime}$ and $\alpha_{s}=120^{\prime}$, and two experimental setups Exp1 and reference experiment. Since the systematic requirements from $\mathrm{B}$-modes detection is sensitive to the inflationary scale in question, it is useful to define an energy scale $E_{\text {crit }}$ below which lensing reconstruction is safe and above which lensing sets the systematic requirements. We define the critical energy $E_{c r i t}$ of a given systematic parameters as the inflationary energy for which the systematic requirements to extract the B-modes signal is equal to 

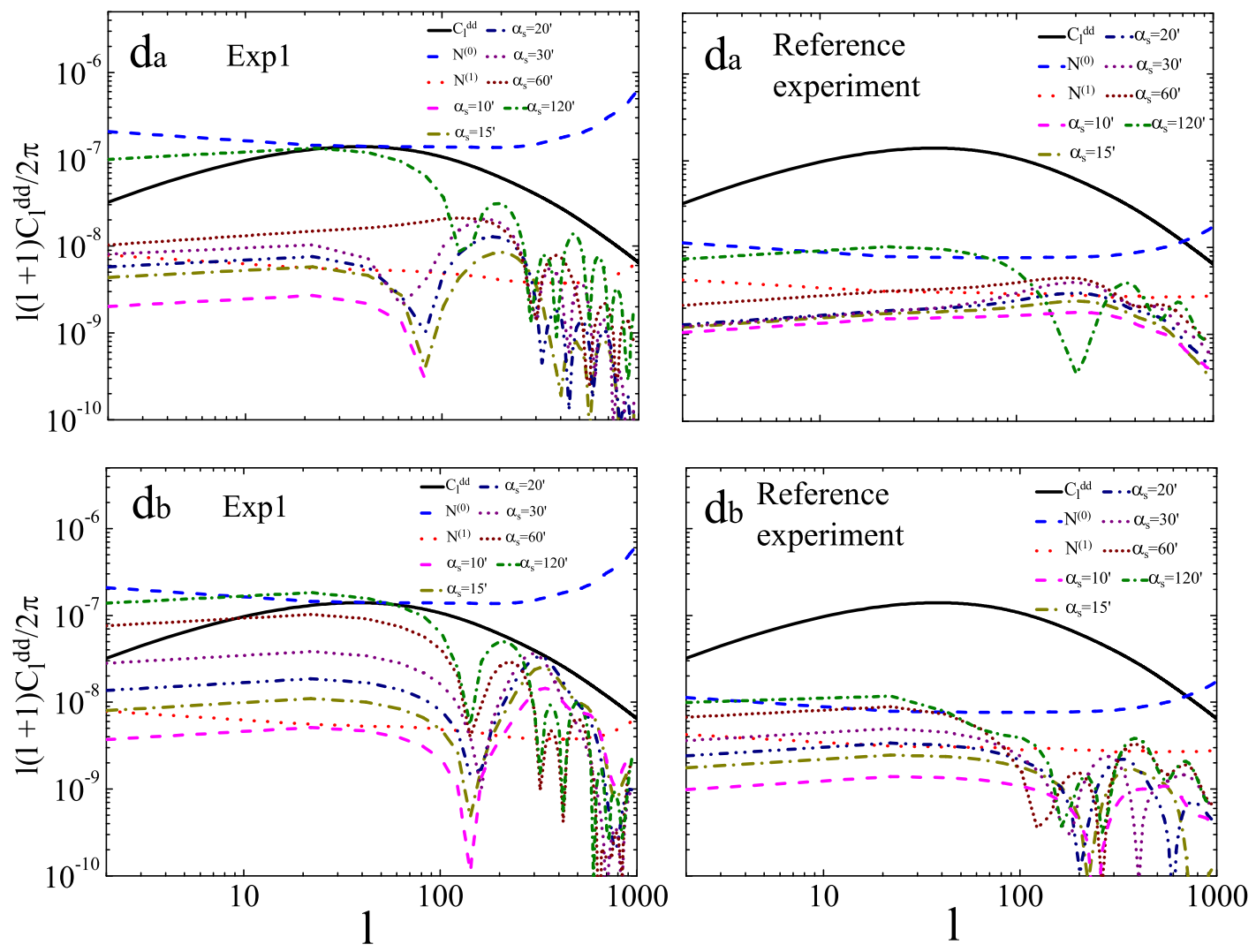

FIG. 5: Upper panels: Contamination from the dipole leakage $d_{a}$ to the deflection angle power spectrum using the EB estimator. The rms fluctuation, $A_{S}$ is assumed to be $10 \%$. The left and right panel are for Exp1 and reference experiment respectively. In both the panels, the solid black, dashed blue, and dot red curves show deflection angle power spectrum $C^{d d}(L)$, Gaussian noise $N^{(0)}(L)$, and the first order non-Gaussian noise $N^{(1)}(L)$. The remaining curves show the absolute value of the systematic bias for various choices of coherence length $\alpha_{s}$, starting from $\alpha_{s}=10^{\prime}$ to $\alpha_{s}=120^{\prime}$. Lower panels: Same as upper panels but for the dipole leakage $d_{b}$.

the requirement to extract the lensing signal. Hence for a given systematic parameter, if the requirement for detecting B-modes with energy scale $E<E_{\text {crit. }}$ have been met, then lensing extraction requirements are already met. Similarly if a given systematic parameter sets the detectable B-modes with energy scale $E>E_{\text {crit., }}$, then lensing extraction requirements provide more stringent constraints on that systematic parameter. We show the critical energy $E_{\text {crit }}$. in Table IV for all the 11 systematic parameters for two choices of coherence length $\left(\alpha_{s}=10^{\prime}\right.$ and $\left.\alpha_{s}=120^{\prime}\right)$ and for both the experimental setups (Exp1 and reference).

For both the Exp1 and reference experiment, lensing reconstruction is safe once the experimental requirements to detect Bmodes with inflationary scale $E_{i}=1.0 \times 10^{16} \mathrm{GeV}$ are met. The requirements from lensing extraction are about $1-2$ orders of magnitude less stringent than the requirements to measure the primordial B-modes with inflationary energy scale of $1.0 \times 10^{16}$ $\mathrm{GeV}$. This means that once the experiment satisfies the requirements of systematic control to detect primordial B-modes, it is safe to use such an experiment to reconstruct the lensing potential power spectrum without extra effort on improving the instrumental systematic control. On the other hand the requirements for lensing reconstruction are comparable or even more stringent for some systematic parameters than the requirements to detect primordial B-modes with inflationary scale $E_{i}=3.0 \times 10^{16} \mathrm{GeV}$.

\section{CONCLUSION}

We illustrate the effects of instrumental systematics on the reconstruction of the projected matter power spectrum from CMB gravitational lensing. We consider seven types of effects which are related to known instrumental systematics: calibration, rotation, pointing, spin-flip, monopole leakage, dipole leakage and quadrupole leakage. These effects can be parametrized by 11 

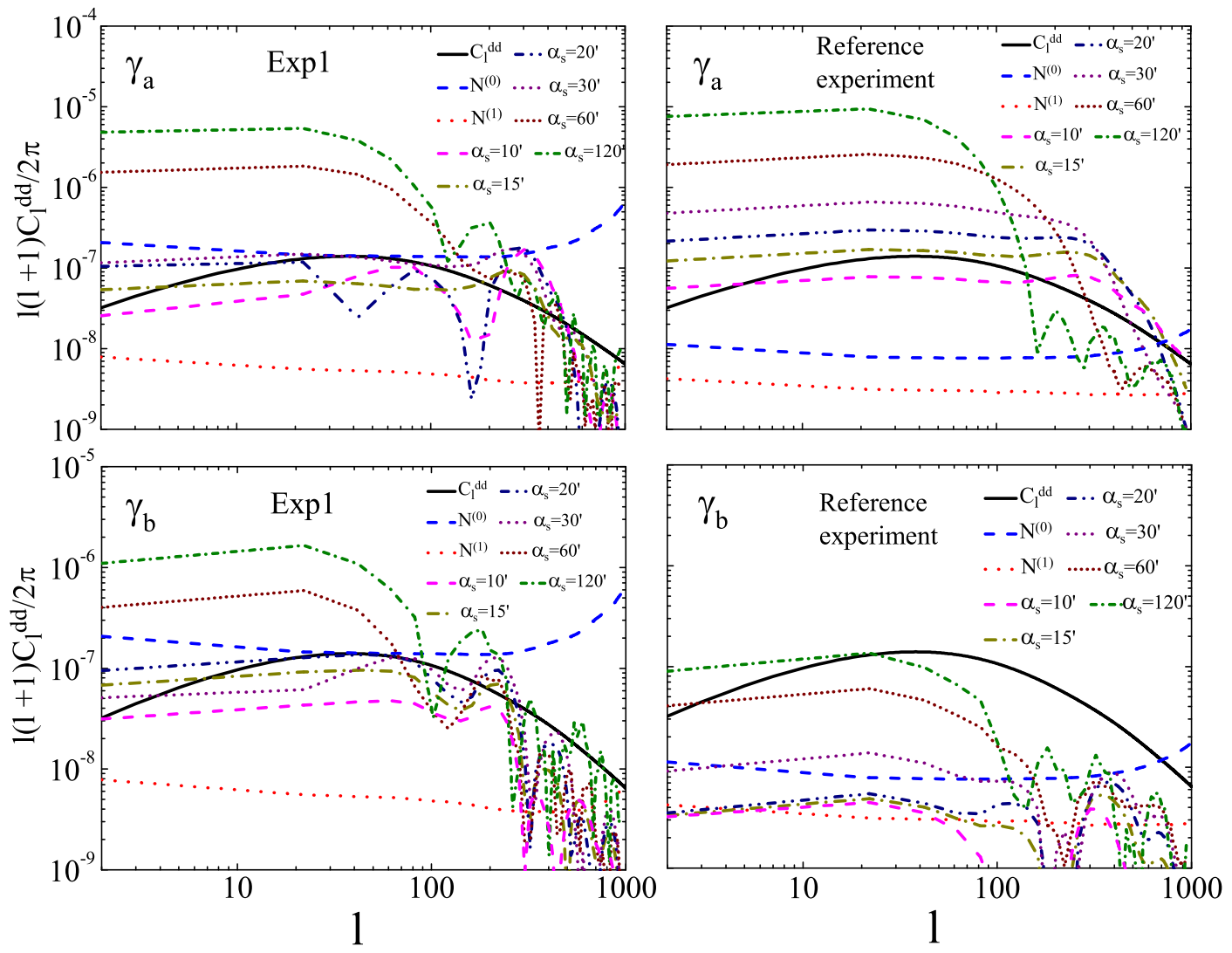

FIG. 6: Upper panels: Contamination from the monopole leakage $\gamma_{a}$ to the deflection angle power spectrum using the EB estimator. The rms fluctuation, $A_{S}$ is assumed to be $10 \%$. The left and right panel are for Exp1 and reference experiment respectively. In both the panels, the solid black, dashed blue, and dot red curves show deflection angle power spectrum $C^{d d}(L)$, Gaussian noise $N^{(0)}(L)$, and the first order non-Gaussian noise $N^{(1)}(L)$. The remaining curves show the absolute value of the systematic bias for various choices of coherence length $\alpha_{s}$, starting from $\alpha_{s}=10^{\prime}$ to $\alpha_{s}=120^{\prime}$. Lower panels: Same as the upper panels but for the monopole leakage $\gamma_{b}$.

distortion fields. Each of these systematic effects can mimic the effective projected matter power spectrum and hence contaminate the lensing reconstruction. We assume a Gaussian distribution for each parameter. We have modeled the fluctuations in the instrumental contamination fields with a coherence length $\left(\alpha_{s}\right)$ and an $r m s$ amplitude $\left(A_{s}\right)$, as defined in Eq. (20) and (21), respectively. Rotation systematics $\omega$ and monopole leakage $\gamma_{a} \& \gamma_{b}$ are among the most dangerous ones.

It is important to know how the systematic effects propagate to the lensing potential reconstruction with good precision, in order to reliably reconstruct the lensing potential. Without well understood and calibrated instrumental systematics, the extraction of cosmological parameters from the statistical properties of lensing potential would also be biased. Systematics in the lensing reconstruction are also one of the key concerns for detecting primordial gravitational waves by removing the contribution properly from lensing-reduced B-modes. A faithful detection of the primordial gravitational wave signal largely depends on how clean the delensing process has been. 


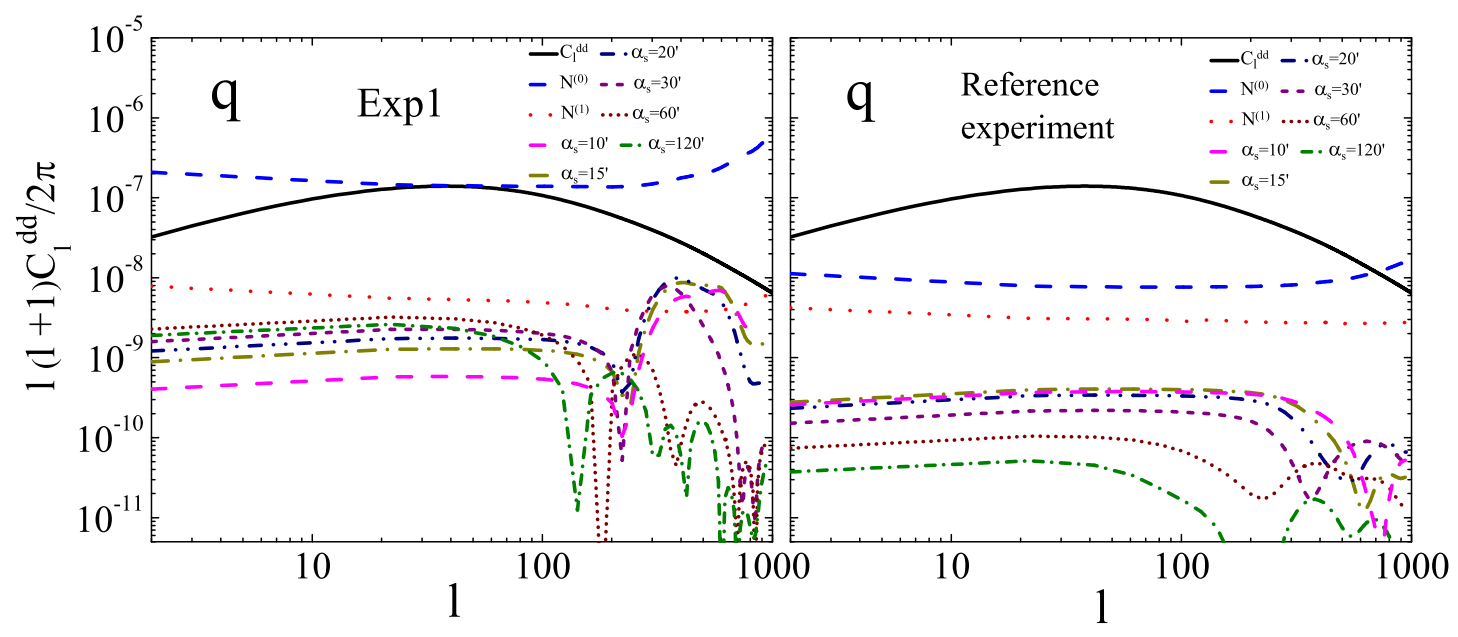

FIG. 7: Contamination from the quadrupole leakage $q$ to the deflection angle power spectrum using the EB estimator. The $r m s$ fluctuation, $A_{S}$ is assumed to be $10 \%$. The left and right panel are for Exp1 and reference experiment respectively. In both the panels, the solid black, dashed blue, and dot red curves show deflection angle power spectrum $C^{d d}(L)$, Gaussian noise $N^{(0)}(L)$, and the first order non-Gaussian noise $N^{(1)}(L)$. The remaining curves show the absolute value of the systematic bias for various choices of coherence length $\alpha_{s}$, starting from $\alpha_{s}=10^{\prime}$ to $\alpha_{s}=120^{\prime}$.

\begin{tabular}{|c|c|c|c|c|c|c|c|c|c|c|c|c|}
\hline \multirow{4}{*}{ Type } & \multicolumn{6}{|c|}{ Exp1 } & \multicolumn{6}{|c|}{ Reference } \\
\hline & \multirow{2}{*}{\multicolumn{2}{|c|}{$\begin{array}{c}\text { Lensing } \\
\ell=40\end{array}$}} & \multicolumn{4}{|c|}{ B-modes } & \multirow{2}{*}{\multicolumn{2}{|c|}{$\begin{array}{c}\text { Lensing } \\
\ell=40\end{array}$}} & \multicolumn{4}{|c|}{ B-modes } \\
\hline & & & \multicolumn{2}{|c|}{$3.0 \times 10^{16} \mathrm{Gev}$} & \multicolumn{2}{|c|}{$1.0 \times 10^{16} \mathrm{GeV}$} & & & $3.0 \times 1$ & ${ }^{16} \mathrm{GeV}$ & $1.0 \times 1$ & $0^{16} \mathrm{GeV}$ \\
\hline & $\alpha_{s}=10^{\prime}$ & $\alpha_{s}=120^{\prime}$ & $\alpha_{s}=10^{\prime}$ & $\alpha_{s}=120^{\prime}$ & $\alpha_{s}=10^{\prime}$ & $\alpha_{s}=120^{\prime}$ & $\alpha_{s}=10^{\prime}$ & $\alpha_{s}=120^{\prime}$ & $\alpha_{s}=10^{\prime}$ & $\alpha_{s}=120^{\prime}$ & $\alpha_{s}=10^{\prime}$ & $\alpha_{s}=120^{\prime}$ \\
\hline Calibration $a$ & 1.04 & 0.55 & 0.549 & 0.468 & 0.061 & 0.052 & 0.86 & 1.56 & 0.486 & 0.468 & 0.054 & 0.052 \\
\hline Rotation $w$ & 0.30 & 0.061 & 0.27 & 0.207 & 0.030 & 0.023 & 0.24 & 0.11 & 0.243 & 0.198 & 0.027 & 0.022 \\
\hline Pointing $p_{a}$ & 2.10 & 1.57 & 8.55 & 6.12 & 0.95 & 0.68 & 1.93 & 5.55 & 15.3 & 12.24 & 1.70 & 1.36 \\
\hline Pointing $p_{b}$ & 1.39 & 2.76 & 1.08 & 6.57 & 0.12 & 0.73 & 2.31 & 9.74 & 1.71 & 13.14 & 0.19 & 1.46 \\
\hline Flip $f_{a}$ & 1.08 & 0.17 & 0.549 & 0.441 & 0.061 & 0.049 & 0.62 & 0.31 & 0.486 & 0.432 & 0.054 & 0.048 \\
\hline Flip $f_{b}$ & 0.61 & 0.17 & 0.531 & 0.360 & 0.059 & 0.040 & 0.58 & 0.31 & 0.477 & 0.36 & 0.053 & 0.040 \\
\hline Monopole $\gamma_{a}$ & 0.114 & 0.024 & 0.021 & 0.0058 & 0.0023 & 0.00064 & 0.13 & 0.013 & 0.0207 & 0.0058 & 0.0023 & 0.00064 \\
\hline Monopole $\gamma_{b}$ & 0.114 & 0.036 & 0.014 & 0.0034 & 0.0016 & 0.00038 & 0.64 & 0.12 & 0.0144 & 0.0034 & 0.0016 & 0.00038 \\
\hline Dipole $d_{a}$ & 0.82 & 0.11 & 0.085 & 0.060 & 0.0094 & 0.0067 & 0.97 & 0.39 & 0.153 & 0.117 & 0.017 & 0.013 \\
\hline Dipole $d_{b}$ & 0.55 & 0.092 & 0.85 & 0.063 & 0.0094 & 0.0070 & 1.02 & 0.33 & 0.153 & 0.126 & 0.017 & 0.014 \\
\hline Quadrupole $q$ & 1.58 & 0.78 & 0.162 & 0.558 & 0.018 & 0.062 & 2.38 & 5.47 & 0.495 & 2.25 & 0.055 & 0.25 \\
\hline
\end{tabular}

TABLE III: Systematic contamination for lensing and B-mode detection for the two experimental setups (Exp1 and reference), for two choices of coherence length $\alpha_{s}=10^{\prime}$ and $\alpha_{s}=120^{\prime}$. We scale the rms amplitude of the systematics field $A_{s}$ to the same level as the maximum of the signal (deflection angle power spectrum at $\ell=40$ and B-mode signal for a fiducial energy scale at $\ell=90$ ). First column mentions the type of systematic parameter in consideration. Within the lensing columns we show the maximum $A_{s}$ (for $\alpha_{s}=10^{\prime}$ and $\alpha_{s}=120^{\prime}$ ) for bias to not exceed the lensing power spectrum. For B-modes columns we show the maximum $A_{s}$ (for $\alpha_{s}=10^{\prime}$ and $\alpha_{s}=120^{\prime}$ ) for bias to not exceed the B-mode signal for two choices of inflationary energy scale $E=3.0 \times 10^{16} \mathrm{GeV}$ and $E=1.0 \times 10^{16} \mathrm{GeV}$ respectively. 


\begin{tabular}{||c|cc||cc||}
\hline \multirow{2}{*}{ Type } & \multicolumn{2}{|c||}{ Exp1 } & \multicolumn{2}{c||}{ Reference } \\
\cline { 2 - 5 } & \multicolumn{2}{|c||}{$E_{\text {crit. }}(\mathrm{GeV})$} & \multicolumn{2}{|c}{$E_{\text {crit. }}(\mathrm{GeV})$} \\
\cline { 2 - 5 } & $\alpha_{s}=10^{\prime}$ & $\alpha_{s}=120^{\prime}$ & $\alpha_{s}=10^{\prime}$ & $\alpha_{s}=120^{\prime}$ \\
\hline Calibration $a$ & 4.13 & 3.25 & 3.99 & 8.83 \\
Rotation $w$ & 3.16 & 1.63 & 2.98 & 2.24 \\
Pointing $p_{a}$ & 1.49 & 1.52 & 1.07 & 2.02 \\
Pointing $p_{b}$ & 3.40 & 1.94 & 3.49 & 2.58 \\
Flip $f_{a}$ & 4.21 & 1.86 & 3.34 & 2.54 \\
Flip $f_{b}$ & 3.22 & 2.06 & 3.31 & 2.78 \\
Monopole $\gamma_{a}$ & 7.80 & 5.00 & 7.80 & 4.33 \\
Monopole $\gamma_{b}$ & 8.29 & 9.73 & 20.00 & 17.77 \\
Dipole $d_{a}$ & 9.34 & 4.05 & 7.55 & 5.48 \\
Dipole $d_{b}$ & 7.51 & 3.57 & 7.78 & 5.00 \\
Quadrupole $q$ & 9.40 & 3.48 & 6.02 & 6.42 \\
\hline
\end{tabular}

TABLE IV: We show the critical inflation energy scale $E_{\text {crit. }}$ for each systematic parameter, for two choices for coherence length $\left(\alpha_{s}=10^{\prime}\right.$ and $\alpha_{s}=120^{\prime}$ ) and for two experimental setups (Exp1 and reference). First column mentions the type of systematic parameter in consideration. $E_{c r i t}$ of a given systematic parameter is the inflationary energy for which the systematic contamination (from that parameter) is equal to both the maximum B-modes signal (corresponding to $\ell=90$ ) and the lensing signal (corresponds to $\ell=40$ ). Hence for a given systematic parameter if the requirement for detecting B-modes with energy scale $E \leq E_{\text {crit. }}$ have been met, then lensing extraction requirement are already met. Similarly if a given systematic parameter sets the detectable B-modes with energy scale $E \geq E_{c r i t}$, then lensing extraction requirement provide more stringent constraint on that systematic parameter.

\section{Acknowledgments}

APSY thanks Daniel Baumann, Ben Wandelt, Scott Kruger, Oliver Zahn, and Jaiyul Yoo for useful discussions and help during the project. We especially thank Daniel Baumann for reading the manuscript and providing useful feedback.

[1] J. M. Kovac, et al., Nature 420, 772 (2002).

[2] D. N. Spergel, et al., Astrophys. J. Suppl. Ser. 170, 377 (2007).

[3] U. Seljak, and M. Zaldarriaga, Phys. Rev. Lett. 78, 2054 (1997); M. Zaldarriaga, and U. Seljak, Phys. Rev. D 55, 1830 (1997).

[4] M. Kamionkowski, A. Kosowsky, A. Stebbins, Phys. Rev. D 55, 7368 (1997); M. Kamionkowski, A. Kosowsky, A. Stebbins, Phys. Rev. Lett. 78, 2058 (1997).

[5] U. Seljak, Astrophys. J. 463, 1 (1996).

[6] M. Zaldarriaga, and U. Seljak, Phys. Rev. D 58, 023003 (1998); U. Seljak, and M. Zaldarriaga, Phys. Rev. Lett. 82 , 2636 (1999); U. Seljak, and M. Zaldarriaga, Phys. Rev. D 60, 043504 (1999);

[7] W. Hu, Phys. Rev. D 62, 043007 (2000).

[8] K. Benabed, F. Bernardeau, and L. Waerbeke, Phys. Rev. D 63, 043501 (2001).

[9] W. Hu, D. Huterer, and K. M. Smith, Astrophys. J. 650, L13 (2006).

[10] K. M. Smith, W. Hu, and M. Kaplinghat, Phys. Rev. D 74, 123002 (2006).

[11] S. Smith, A. Challinor, and G. Rocha, Phys. Rev. D 73, 023517 (2006). (2006).

[12] V. Acquaviva, and C. Baccigalupi, Phys. Rev. D 74, 103510 (2006).

[13] E. Calabrese, et. al, Phys. Rev. D 77, 123531 (2008).

[14] D. Sarkar, P. Serra, A. Cooray, K. Ichiki, and D. Baumann, Phys. Rev. D 77, 103515 (2008).

[15] W. Hu, Astrophys. J., 557, L79 (2001); W. Hu, and T. Okamoto, Astrophys. J. 574, 566 (2002); T. Okamoto, and W. Hu, Phys. Rev. D 67, 083002 (2003).

[16] M. Zaldarriaga, and U. Seljak, Phys. Rev. D 59, 123507 (1999); M. Zaldarriaga, Phys. Rev. D 62, 063510 (2000).

[17] J. Guzik, U. Seljak, and M. Zaldarriaga, Phys. Rev. D 62, 043517 (2000).

[18] C. M. Hirata, and U. Seljak, Phys. Rev. D 67, 043001 (2003); C. M. Hirata, and U. Seljak, Phys. Rev. D 68, 083002 (2003).

[19] U. Seljak, and C. M. Hirata, Phys. Rev. D 69, 043005 (2004).

[20] M. Kesden, A. Cooray, and M. Kamionkowski, Phys. Rev. Lett. 89, 011304 (2002).

[21] M. Kesden, A. Cooray, and M. Kamionkowski, Phys. Rev. D 67, 123507 (2003).

[22] A. Cooray, Phys. Rev. D 65, 063512 (2002); A. Cooray, and M. Kesden, New Astron. 8, 231 (2003); A. Cooray, Phys. Rev. D 66, 103509 (2002).

[23] W. Hu, Phys. Rev. D 65, 023003 (2002). 
[24] http://cmbpol.uchicago.edu/; D. Baumann, et al., arXiv:0811.3911; K. M. Smith, et al., arXiv:0811.3916, M. Zaldarriaga, et al., arXiv:0811.3918, D. Baumann, et al., arXiv:0811.3919, J. Dunkley, et al., arXiv:0811.3915, A. A. Fraisse, et al., arXiv:0811.3920

[25] P. Oxley et al., Proc. SPIEInt. Soc. Opt. Eng., 5543, 320 (2004), arXiv:astro-ph/0501111, http://groups.physics.umn.edu/cosmology/ebex/

[26] C. J. MacTavish, et al., arXiv:0710.0375; B. P. Crill, et al. arXiv 0807.1548.

[27] J.A. Rubiño-Martín, et al., arXiv:0810.3141.

[28] J. Bock, et al., arXiv:astro-ph/0604101

[29] J. Bock, et al., arXiv:0805.4207.

[30] C. Bischoff, et al. Astrophys. J. 684, 771 (2008).

[31] L. Verde, H. Peiris, and R. Jimenez, JCAP, 0601, 019 (2006); M. Kaplinghat, New Astronomy Reviews, 47, 893 (2003).

[32] W. Hu, M. M. Hedman, \& M. Zaldarriaga, Phys. Rev. D, 67, 043004 (2003).

[33] D. O’Dea, A. Challinor, \& B. R. Johnson, MNRAS, 376, 1767 (2007).

[34] M. Shimon, B. Keating, N. Ponthieu, and E. Hivon, Phys. Rev. D 77, 083003 (2008).

[35] N. J. Miller, M. Shimon, B. G. Keating, arXiv:0806.3096

[36] E. F. Bunn, Phys. Rev. D 75, 083517 (2007).

[37] C. Rosset, et al. A\&A, 464, 405 (2006).

[38] M. L. Brown, et al., arXiv:0809.4032

[39] M. A. J. Ashdown, et al., arXiv:0806.3167

[40] U. Seljak, and M. Zaldarriaga, Astrophys. J. 469, 437 (1996).

[41] A. Lewis, and A. Challinor, Phys. Rept. 429, 1 (2006).

[42] R. A. Sunyaev, and Ya. B. Zel'dovich, MNRAS, 190, 413 (1980).

[43] R. K. Sachs, and A. M. Wolfe, Astrophys. J. 147, 73 (1967).

[44] M. A. Riquelme, and D. N. Spergel, Astrophys. J. 661, 672 (2007).

[45] J. Lesgourgues, M. Liguori, S. Matarrese, and A. Riotto, Phys. Rev. D 71, 103514 (2005).

[46] T. Okamoto, and W. Hu, Phys. Rev. D 66, 063008 (2002); W. Hu, Phys. Rev. D 64, 083005 (2001).

[47] A. Challinor, and A. Lewis, Phys. Rev. D 71, 103010 (2005).

\section{APPENDIX A: INSTRUMENTAL SYSTEMATICS FOR THE TT ESTIMATOR}

In this appendix, we show detailed calculation of instrumental systematic contamination on the TT estimator. The calculation is similar to the systematic contamination analysis for other estimators involving the polarization fields (discussed in Appendix B.

We first expand the observed temperature $\tilde{T}^{o b s}(\hat{\mathbf{n}})$ field, given by in Eq. (13) as

$$
\begin{aligned}
\tilde{T}^{o b s}(\hat{\mathbf{n}})= & {[1+a(\hat{\mathbf{n}})] T[\hat{\mathbf{n}}+\nabla \phi(\hat{\mathbf{n}})] } \\
\approx & T(\hat{\mathbf{n}})+\nabla_{a} \phi(\hat{\mathbf{n}}) \nabla^{a} T(\hat{\mathbf{n}})+\frac{1}{2} \nabla_{a} \phi(\hat{\mathbf{n}}) \nabla_{b} \phi(\hat{\mathbf{n}}) \nabla^{a} \nabla^{b} T(\hat{\mathbf{n}})+\ldots \\
& \quad+a(\hat{\mathbf{n}}) \times\left[T(\hat{\mathbf{n}})+\nabla_{a} \phi(\hat{\mathbf{n}}) \nabla^{a} T(\hat{\mathbf{n}})+\frac{1}{2} \nabla_{a} \phi(\hat{\mathbf{n}}) \nabla_{b} \phi(\hat{\mathbf{n}}) \nabla^{a} \nabla^{b} T(\hat{\mathbf{n}})+\ldots\right],
\end{aligned}
$$

where $a(\hat{\mathbf{n}})$ is the calibration parameter. Taking the Fourier transform of the lensed map with systematics $\tilde{T}^{\text {obs }}(\hat{\mathbf{n}})$ under the flat-sky approximation,

$$
\begin{aligned}
\tilde{T}^{\mathrm{obs}}(\mathbf{l}) & =\int d \hat{\mathbf{n}}[1+a(\hat{\mathbf{n}})] \tilde{T}(\hat{\mathbf{n}}) e^{-i \mathbf{l} \cdot \hat{\mathbf{n}}} \\
& =T(\mathbf{l})-\int \frac{d^{2} \mathbf{l}^{\prime}}{(2 \pi)^{2}} T\left(\mathbf{l}^{\prime}\right) L\left(\mathbf{l}, \mathbf{l}^{\prime}\right),
\end{aligned}
$$

where

$$
\begin{aligned}
& L\left(\mathbf{l}, \mathbf{l}^{\prime}\right) \equiv \phi\left(\mathbf{l}-\mathbf{l}^{\prime}\right)\left[\left(\mathbf{l}-\mathbf{l}^{\prime}\right) \cdot \mathbf{l}^{\prime}\right]+\frac{1}{2} \int \frac{d^{2} \mathbf{l}^{\prime \prime}}{(2 \pi)^{2}} \phi\left(\mathbf{l}^{\prime \prime}\right) \times \phi\left(\mathbf{l}-\mathbf{l}^{\prime}-\mathbf{l}^{\prime \prime}\right)\left(\mathbf{l}^{\prime \prime} \cdot \mathbf{l}^{\prime}\right)\left[\left(\mathbf{l}^{\prime \prime}+\mathbf{l}^{\prime}-\mathbf{l}\right) \cdot \mathbf{l}^{\prime}\right]+\ldots \\
&-a\left(\mathbf{l}-\mathbf{l}^{\prime}\right)+\int \frac{d^{2} \mathbf{l}^{\prime \prime}}{(2 \pi)^{2}} a\left(\mathbf{l}^{\prime \prime}\right) \times\left[\mathbf{l}^{\prime} \cdot\left(\mathbf{l}-\mathbf{l}^{\prime}-\mathbf{l}^{\prime \prime}\right)\right] \phi\left(\mathbf{l}-\mathbf{l}^{\prime}-\mathbf{l}^{\prime \prime}\right) \\
&+\frac{1}{2} \int \frac{d^{2} \mathbf{l}^{\prime \prime}}{(2 \pi)^{2}} \int \frac{d^{2} \mathbf{l}^{\prime \prime \prime}}{(2 \pi)^{2}} a\left(\mathbf{l}^{\prime \prime \prime}\right) \times \phi\left(\mathbf{l}^{\prime \prime}\right) \times \phi\left(\mathbf{l}-\mathbf{l}^{\prime}-\mathbf{l}^{\prime \prime}-\mathbf{l}^{\prime \prime \prime}\right)\left(\mathbf{l}^{\prime \prime} \cdot \mathbf{l}^{\prime}\right)\left[\left(\mathbf{l}^{\prime \prime \prime}+\mathbf{l}^{\prime \prime}+\mathbf{l}^{\prime}-\mathbf{l}\right) \cdot \mathbf{l}^{\prime}\right]+\ldots
\end{aligned}
$$

Using the above equations one can compute the CMB temperature power spectrum $C_{l}^{T T}$ in presence of systematic contamination, the result is given in Eq. (14). 
Analysis of the deflection angle power spectrum $C_{l}^{d d}$ involves trispectrum calculation. Below we show the details of the temperature trispectrum including the systematic contribution,

$$
\begin{aligned}
& \left.\left\langle T^{o b s}\left(\mathbf{l}_{1}\right) T^{o b s}\left(\mathbf{l}_{2}\right) T^{o b s}\left(\mathbf{l}_{1}{ }^{\prime}\right) T^{o b s} \mathbf{l}_{2}{ }^{\prime}\right)\right\rangle_{\mathrm{CMB}}= \\
& \left\{\left(C_{l_{1}}^{T T}+C_{l_{1}}^{T T \mathrm{n}}\right)(2 \pi)^{2} \delta_{\mathrm{D}}(\mathbf{L})+a(\mathbf{L})\left(C_{l_{1}}^{T T}+C_{l_{2}}^{T T}\right)+\phi(\mathbf{L}) f_{T T}\left(\mathbf{l}_{1}, \mathbf{l}_{2}\right)-\int \frac{d^{2} \mathbf{l}_{1}^{\prime}}{(2 \pi)^{2}} a\left(\mathbf{l}_{1}{ }^{\prime}\right)\left(\mathbf{L}-\mathbf{l}_{1}{ }^{\prime}\right) \phi\left(\mathbf{L}-\mathbf{l}_{1}{ }^{\prime}\right) \mathbf{l}_{2} C_{l_{2}}^{T T}\right. \\
& -\int \frac{d^{2} \mathbf{l}_{2}^{\prime}}{(2 \pi)^{2}} a\left(\mathbf{l}_{2}{ }^{\prime}\right)\left(\mathbf{L}-\mathbf{l}_{2}{ }^{\prime}\right) \phi\left(\mathbf{L}-\mathbf{l}_{2}{ }^{\prime}\right) \mathbf{l}_{1} C_{l_{1}}^{T T}+\frac{1}{2} \int \frac{d^{2} \mathbf{l}_{1}^{\prime}}{(2 \pi)^{2}} \phi\left(\mathbf{l}_{1}{ }^{\prime}\right) \phi\left(\mathbf{L}-\mathbf{l}_{1}{ }^{\prime}\right)\left\{C_{l_{1}}^{T T}\left(\mathbf{l}_{1} \cdot \mathbf{l}_{1}{ }^{\prime}\right)\left[\mathbf{l}_{1} \cdot\left(\mathbf{L}-\mathbf{l}_{1}{ }^{\prime}\right)\right]+C_{l_{2}}^{T T}\left(\mathbf{l}_{2} \cdot \mathbf{l}_{1}{ }^{\prime}\right)\left[\mathbf{l}_{2} \cdot\left(\mathbf{L}-\mathbf{l}_{1}{ }^{\prime}\right)\right]\right\} \\
& +\int \frac{d^{2} \mathbf{l}_{1}^{\prime}}{(2 \pi)^{2}} a\left(\mathbf{l}_{1}-\mathbf{l}_{1}{ }^{\prime}\right) a\left(\mathbf{l}_{2}+\mathbf{l}_{1}{ }^{\prime}\right) C_{l_{1}^{\prime}}^{T T}-\int \frac{d^{2} \mathbf{l}_{1}^{\prime}}{(2 \pi)^{2}} C_{l_{1}^{\prime}}^{T T} \phi\left(\mathbf{l}_{1}-\mathbf{l}_{1}{ }^{\prime}\right) \phi\left(\mathbf{l}_{2}+\mathbf{l}_{1}{ }^{\prime}\right)\left[\mathbf{l}_{1}{ }^{\prime} \cdot\left(\mathbf{l}_{1}-\mathbf{l}_{1}{ }^{\prime}\right)\right] \times\left[\mathbf{l}_{1}{ }^{\prime} \cdot\left(\mathbf{l}_{2}+\mathbf{l}_{1}{ }^{\prime}\right)\right] \\
& \left.-\int \frac{d^{2} \mathbf{l}_{2}^{\prime}}{(2 \pi)^{2}} C_{l_{2}^{\prime}}^{T T} \phi\left(\mathbf{l}_{2}-\mathbf{l}_{2}{ }^{\prime}\right) a\left(\mathbf{l}_{1}+\mathbf{l}_{2}{ }^{\prime}\right)\left[\mathbf{l}_{2}{ }^{\prime} \cdot\left(\mathbf{l}_{2}-\mathbf{l}_{2}{ }^{\prime}\right)\right]-\int \frac{d^{2} \mathbf{l}_{1}^{\prime}}{(2 \pi)^{2}} C_{l_{1}^{\prime}}^{T T} \phi\left(\mathbf{l}_{1}-\mathbf{l}_{1}{ }^{\prime}\right) a\left(\mathbf{l}_{2}+\mathbf{l}_{1}{ }^{\prime}\right)\left[\mathbf{l}_{1}{ }^{\prime} \cdot\left(\mathbf{l}_{1}-\mathbf{l}_{1}{ }^{\prime}\right)\right]\right\} \\
& \times\left\{\left(C_{l_{1}^{\prime}}^{T T}+C_{l_{1}^{\prime}}^{T T \mathrm{n}}\right)(2 \pi)^{2} \delta_{\mathrm{D}}(\mathbf{L})+a(\mathbf{L})\left(C_{l_{1}^{\prime}}^{T T}+C_{l_{2}^{\prime}}^{T T}\right)+\phi(\mathbf{L}) f_{T T}\left(\mathbf{l}_{1}{ }^{\prime}, \mathbf{l}_{2}{ }^{\prime}\right)-\int \frac{d^{2} \mathbf{l}}{(2 \pi)^{2}} a(\mathbf{l})(\mathbf{L}-\mathbf{l}) \phi(\mathbf{L}-\mathbf{l}) \mathbf{l}_{2}{ }^{\prime} C_{l_{2}^{\prime}}^{T T}\right. \\
& -\int \frac{d^{2} \mathbf{l}}{(2 \pi)^{2}} a(\mathbf{l})(\mathbf{L}-\mathbf{l}) \phi(\mathbf{L}-\mathbf{l}) \mathbf{l}_{1}{ }^{\prime} C_{l_{1}^{\prime}}^{T T}+\frac{1}{2} \int \frac{d^{2} \mathbf{l}}{(2 \pi)^{2}} \phi(\mathbf{l}) \phi(\mathbf{L}-\mathbf{l})\left\{C_{l_{1}^{\prime}}^{T T}\left(\mathbf{l}_{1}{ }^{\prime} \cdot \mathbf{l}\right)\left[\mathbf{l}_{1}{ }^{\prime} \cdot(\mathbf{L}-\mathbf{l})\right]+C_{l_{2}^{\prime}}^{T T}\left(\mathbf{l}_{2}{ }^{\prime} \cdot \mathbf{l}\right)\left[\mathbf{l}_{2}{ }^{\prime} \cdot(\mathbf{L}-\mathbf{l})\right]\right\} \\
& +\int \frac{d^{2} \mathbf{l}}{(2 \pi)^{2}} a\left(\mathbf{l}_{1}{ }^{\prime}-\mathbf{l}\right) a\left(\mathbf{l}_{2}{ }^{\prime}+\mathbf{l}\right) C_{l}^{T T}-\int \frac{d^{2} \mathbf{l}}{(2 \pi)^{2}} C_{l}^{T T} \phi\left(\mathbf{l}_{1}{ }^{\prime}-\mathbf{l}\right) \phi\left(\mathbf{l}_{2}{ }^{\prime}+\mathbf{l}\right)\left[\mathbf{l} \cdot\left(\mathbf{l}_{1}{ }^{\prime}-\mathbf{l}\right)\right] \times\left[\mathbf{l} \cdot\left(\mathbf{l}_{2}{ }^{\prime}+\mathbf{l}\right)\right] \\
& \left.-\int \frac{d^{2} \mathbf{l}}{(2 \pi)^{2}} C_{l}^{T T} \phi\left(\mathbf{l}_{2}{ }^{\prime}-\mathbf{l}\right) a\left(\mathbf{l}_{1}{ }^{\prime}+\mathbf{l}\right)\left[\mathbf{l} \cdot\left(\mathbf{l}_{2}{ }^{\prime}-\mathbf{l}\right)\right]-\int \frac{d^{2} \mathbf{l}}{(2 \pi)^{2}} C_{l}^{T T} \phi\left(\mathbf{l}_{1}{ }^{\prime}-\mathbf{l}\right) a\left(\mathbf{l}_{2}{ }^{\prime}+\mathbf{l}\right)\left[\mathbf{l} \cdot\left(\mathbf{l}_{1}{ }^{\prime}-\mathbf{l}\right)\right]\right\}+ \text { Perm. }
\end{aligned}
$$

In practice, we do not know the large-scale structure between us and the last-scattering surface, we must average over different realizations of the large-scale structure (denoted by \langle\rangle$_{\text {LSS }}$ ) and also over different realizations of the systematic fields (denoted by \langle\rangle$\left._{\text {SYS }}\right)$ to obtain the observed deflection angle power spectrum. The deflection field power spectrum can be estimated by the TT estimator as

$$
\begin{aligned}
\left\langle\left\langle\left\langle d_{T T}(\mathbf{L}) \cdot d_{T T}\left(\mathbf{L}^{\prime}\right)\right\rangle_{\mathrm{CMB}}\right\rangle_{\mathrm{LSS}}\right\rangle_{\mathrm{SYS}} \equiv & \left\langle d_{T T}(\mathbf{L}) \cdot d_{T T}\left(\mathbf{L}^{\prime}\right)\right\rangle \\
= & \left(\mathbf{L} \cdot \mathbf{L}^{\prime}\right) \frac{A_{T T}(L)}{L^{2}} \frac{A_{T T}\left(L^{\prime}\right)}{L^{\prime 2}} \\
& \times \int \frac{d^{2} \mathbf{l}_{1}}{(2 \pi)^{2}} \int \frac{d^{2} \mathbf{l}_{1}^{\prime}}{(2 \pi)^{2}}\left\langle\left\langle\left\langle T^{\mathrm{obs}}\left(\mathbf{l}_{1}\right) T^{\mathrm{obs}}\left(\mathbf{l}_{2}\right) T^{\mathrm{obs}}\left(\mathbf{l}_{1}{ }^{\prime}\right) T^{\mathrm{obs}}\left(\mathbf{l}_{2}{ }^{\prime}\right)\right\rangle_{\mathrm{CMB}}\right\rangle_{\mathrm{LSS}}\right\rangle_{\mathrm{SYS}} \\
& \times F_{T T}\left(\mathbf{l}_{1}, \mathbf{l}_{2}\right) F_{T T}\left(\mathbf{l}_{1}{ }^{\prime}, \mathbf{l}_{2}{ }^{\prime}\right) .
\end{aligned}
$$

Only the connected part of the trispectrum comes into the deflection angle power spectrum $C_{\ell}^{d d}$. Keeping the first order of $C_{l}^{\phi \phi}$ and $C_{l}^{a a}$, the results can be simplified as

$$
\begin{aligned}
\left\langle T^{o b s}\left(\mathbf{l}_{1}\right) T^{o b s}\left(\mathbf{l}_{2}\right) T^{o b s}\left(\mathbf{l}_{1}{ }^{\prime}\right) T^{o b s}\left(\mathbf{l}_{2}{ }^{\prime}\right)\right\rangle_{\mathrm{CMB}, \mathrm{conn} .}=\left\langle\int\right. & \frac{d^{2} \mathbf{l}_{1}^{\prime}}{(2 \pi)^{2}} T\left(\mathbf{l}_{1}{ }^{\prime}\right) \phi\left(\mathbf{l}_{1}-\mathbf{l}_{1}{ }^{\prime}\right)\left[\left(\mathbf{l}_{1}-\mathbf{l}_{1}{ }^{\prime}\right) \cdot \mathbf{l}_{1}{ }^{\prime}\right] \\
& \left.\times \int \frac{d^{2} \mathbf{l}_{2}^{\prime}}{(2 \pi)^{2}} T\left(\mathbf{l}_{2}{ }^{\prime}\right) \phi\left(\mathbf{l}_{2}-\mathbf{l}_{2}{ }^{\prime}\right)\left[\left(\mathbf{l}_{2}-\mathbf{l}_{2}{ }^{\prime}\right) \cdot \mathbf{l}_{2}{ }^{\prime}\right] T\left(\mathbf{l}_{1}{ }^{\prime}\right) T\left(\mathbf{l}_{2}{ }^{\prime}\right)\right\rangle \\
& +\left\langle\int \frac{d^{2} \mathbf{l}_{1}^{\prime}}{(2 \pi)^{2}} T\left(\mathbf{l}_{1}{ }^{\prime}\right) a\left(\mathbf{l}_{1}-\mathbf{l}_{1}{ }^{\prime}\right) \int \frac{d^{2} \mathbf{l}_{2}^{\prime}}{(2 \pi)^{2}} T\left(\mathbf{l}_{2}{ }^{\prime}\right) a\left(\mathbf{l}_{2}-\mathbf{l}_{2}{ }^{\prime}\right) T\left(\mathbf{l}_{1}{ }^{\prime}\right) T\left(\mathbf{l}_{2}{ }^{\prime}\right)\right\rangle \\
=C_{l_{1}^{\prime}}^{T} & C_{l_{2}^{\prime}}^{T}\left\langle\phi\left(\mathbf{l}_{1}+\mathbf{l}_{1}{ }^{\prime}\right) \phi\left(\mathbf{l}_{2}+\mathbf{l}_{2}{ }^{\prime}\right)\right\rangle\left[\left(\mathbf{l}_{1}+\mathbf{l}_{1}{ }^{\prime}\right) \cdot \mathbf{l}_{1}{ }^{\prime}\right]\left[\left(\mathbf{l}_{2}+\mathbf{l}_{2}{ }^{\prime}\right) \cdot \mathbf{l}_{2}{ }^{\prime}\right] \\
& +C_{l_{1}^{\prime}}^{T} C_{l_{2}^{\prime}}^{T}\left\langle a\left(\mathbf{l}_{1}+\mathbf{l}_{1}{ }^{\prime}\right) a\left(\mathbf{l}_{2}+\mathbf{l}_{2}{ }^{\prime}\right)\right\rangle \\
& +C_{l_{1}^{\prime}}^{T} C_{l_{2}^{\prime}}^{T}\left\langle\phi\left(\mathbf{l}_{1}+\mathbf{l}_{2}{ }^{\prime}\right) \phi\left(\mathbf{l}_{2}+\mathbf{l}_{1}{ }^{\prime}\right)\right\rangle\left[\left(\mathbf{l}_{1}+\mathbf{l}_{2}{ }^{\prime}\right) \cdot \mathbf{l}_{2}{ }^{\prime}\right]\left[\left(\mathbf{l}_{2}+\mathbf{l}_{1}{ }^{\prime}\right) \cdot \mathbf{l}_{1}{ }^{\prime}\right] \\
& +C_{l_{1}^{\prime}}^{T} C_{l_{2}^{\prime}}^{T}\left\langle a\left(\mathbf{l}_{1}+\mathbf{l}_{2}{ }^{\prime}\right) a\left(\mathbf{l}_{2}+\mathbf{l}_{1}{ }^{\prime}\right)\right\rangle+P e r m .
\end{aligned}
$$

The terms that are linear in the lensing potential $\phi(\mathbf{l})$ and the temperature systematics $a(\mathbf{l})$ vanish. Further averaging over the lensing field and the systematics field, one can get Eq. (16) which gives the measured lensing induced CMB temperature trispectrum in the presence of instrumental systematic contaminations. 


\section{APPENDIX B: POLARIZATION SYSTEMATICS CONTAMINATION}

In this Appendix we calculate the window functions (given in Table @) for each instrumental systematic. We show how the systematic contamination comes in the analysis inevitably. Again the calculation is done for small deflection angles (weak lensing).

We will first do the calculation for the polarization transfer systematics. The Taylor expansion of two Stokes polarization parameters including the systematic parameters can be written as:

$$
\begin{aligned}
{[\tilde{Q} \pm i \tilde{U}]^{o b s}(\hat{\mathbf{n}})=} & {[1+a \pm i 2 \omega](\hat{\mathbf{n}})[Q \pm i U][\hat{\mathbf{n}}+\nabla \phi(\hat{\mathbf{n}})]+\left[f_{1} \pm i f_{2}\right](\hat{\mathbf{n}})[Q \mp i U][\hat{\mathbf{n}}+\nabla \phi(\hat{\mathbf{n}})]+\left[\gamma_{1} \pm i \gamma_{2}\right](\hat{\mathbf{n}}) T[\hat{\mathbf{n}}+\nabla \phi(\hat{\mathbf{n}})] } \\
= & {\left.[Q \pm i U](\hat{\mathbf{n}})+\nabla_{a} \phi(\hat{\mathbf{n}}) \nabla^{a}[Q \pm i U](\hat{\mathbf{n}})+\frac{1}{2} \nabla_{a} \phi(\hat{\mathbf{n}}) \nabla_{b} \phi(\hat{\mathbf{n}}) \nabla^{a} \nabla^{b}[Q \pm i U](\hat{\mathbf{n}})\right]+\ldots } \\
& +[a \pm i 2 \omega](\hat{\mathbf{n}})\left[[Q \pm i U](\hat{\mathbf{n}})+\nabla_{a} \phi(\hat{\mathbf{n}}) \nabla^{a}[Q \pm i U](\hat{\mathbf{n}})+\frac{1}{2} \nabla_{a} \phi(\hat{\mathbf{n}}) \nabla_{b} \phi(\hat{\mathbf{n}}) \nabla^{a} \nabla^{b}[Q \pm i U](\hat{\mathbf{n}})+\ldots\right] \\
& \left.+\left[f_{1} \pm i f_{2}\right](\hat{\mathbf{n}})\left[[Q \mp i U](\hat{\mathbf{n}})+\nabla_{a} \phi(\hat{\mathbf{n}}) \nabla^{a}[Q \mp i U](\hat{\mathbf{n}})+\frac{1}{2} \nabla_{a} \phi(\hat{\mathbf{n}}) \nabla_{b} \phi(\hat{\mathbf{n}}) \nabla^{a} \nabla^{b}[Q \mp i U](\hat{\mathbf{n}})+\ldots\right]\right] \\
& +\left[\gamma_{1} \pm i \gamma_{2}\right](\hat{\mathbf{n}})\left[T(\hat{\mathbf{n}})+\nabla_{a} \phi(\hat{\mathbf{n}}) \nabla^{a} T(\hat{\mathbf{n}})+\frac{1}{2} \nabla_{a} \phi(\hat{\mathbf{n}}) \nabla_{b} \phi(\hat{\mathbf{n}}) \nabla^{a} \nabla^{b} T(\hat{\mathbf{n}})+\ldots\right] .
\end{aligned}
$$

Performing the harmonic transformation we get

$$
\begin{aligned}
{[\tilde{E}(\mathbf{l}) \pm i \tilde{B}(\mathbf{l})]^{o b s} } & =\int d \hat{\mathbf{n}}[\tilde{Q} \pm i \tilde{U}]^{o b s}(\hat{\mathbf{n}}) e^{\mp 2 i \varphi \mathbf{l}} e^{-i \mathbf{l} \cdot \hat{\mathbf{n}}} \\
& =E(\mathbf{l}) \pm i B(\mathbf{l})-\int \frac{d^{2} \mathbf{l}^{\prime}}{(2 \pi)^{2}}\left[E\left(\mathbf{l}^{\prime}\right) \pm i B\left(\mathbf{l}^{\prime}\right)\right] L_{P}\left(\mathbf{l}, \mathbf{l}^{\prime}\right)-\int \frac{d^{2} \mathbf{l}^{\prime}}{(2 \pi)^{2}} T\left(\mathbf{l}^{\prime}\right) L_{T}\left(\mathbf{l}, \mathbf{l}^{\prime}\right),
\end{aligned}
$$

where

$$
\begin{aligned}
& L_{P}\left(\mathbf{l}, \mathbf{l}^{\prime}\right) \equiv e^{ \pm 2 i\left(\varphi_{\mathbf{l}^{\prime}}-\varphi_{\mathbf{l}}\right)} \phi\left(\mathbf{l}-\mathbf{l}^{\prime}\right)\left[\left(\mathbf{l}-\mathbf{l}^{\prime}\right) \cdot \mathbf{l}^{\prime}\right]+\frac{1}{2} \int \frac{d^{2} \mathbf{l}^{\prime \prime}}{(2 \pi)^{2}} e^{ \pm 2 i\left(\varphi_{\mathbf{l}^{\prime}}-\varphi_{1}\right)} \phi\left(\mathbf{l}^{\prime \prime}\right) \times \phi\left(\mathbf{l}-\mathbf{l}^{\prime}-\mathbf{l}^{\prime \prime}\right)\left(\mathbf{l}^{\prime \prime} \cdot \mathbf{l}^{\prime}\right)\left[\left(\mathbf{l}^{\prime \prime}+\mathbf{l}^{\prime}-\mathbf{l}\right) \cdot \mathbf{l}^{\prime}\right]+\ldots \\
& -e^{ \pm 2 i\left(\varphi_{\mathbf{l}^{\prime}}-\varphi_{1}\right)}\left[a\left(\mathbf{l}-\mathbf{l}^{\prime}\right) \pm i 2 \omega\left(\mathbf{l}-\mathbf{l}^{\prime}\right)\right]+\int \frac{d^{2} \mathbf{l}^{\prime \prime}}{(2 \pi)^{2}} e^{ \pm 2 i\left(\varphi_{\mathbf{l}^{\prime}}-\varphi_{\mathbf{l}}\right)}\left[a\left(\mathbf{l}^{\prime \prime}\right) \pm i 2 \omega\left(\mathbf{l}^{\prime \prime}\right)\right] \times\left[\mathbf{l}^{\prime} \cdot\left(\mathbf{l}-\mathbf{l}^{\prime}-\mathbf{l}^{\prime \prime}\right)\right] \phi\left(\mathbf{l}-\mathbf{l}^{\prime}-\mathbf{l}^{\prime \prime}\right) \ldots \\
& -e^{ \pm 2 i\left(2 \varphi_{1-\mathbf{I}^{\prime}}-\varphi_{\mathbf{1}^{\prime}}-\varphi_{\mathbf{1}}\right)}\left[f_{1}\left(\mathbf{l}-\mathbf{l}^{\prime}\right) \pm i f_{2}\left(\mathbf{l}-\mathbf{l}^{\prime}\right)\right]+\int \frac{d^{2} \mathbf{l}^{\prime \prime}}{(2 \pi)^{2}} e^{ \pm 2 i\left(2 \varphi_{\mathbf{1}^{\prime \prime}}-\varphi_{\mathbf{1}^{\prime}}-\varphi_{\mathbf{1}}\right)}\left[f_{1}\left(\mathbf{l}^{\prime \prime}\right) \pm i f_{2}\left(\mathbf{l}^{\prime \prime}\right)\right] \times\left[\mathbf{l}^{\prime} \cdot\left(\mathbf{l}-\mathbf{l}^{\prime}-\mathbf{l}^{\prime \prime}\right)\right] \phi\left(\mathbf{l}-\mathbf{l}^{\prime}-\mathbf{l}^{\prime \prime}\right) \ldots
\end{aligned}
$$

and

$$
L_{T}\left(\mathbf{l}, \mathbf{l}^{\prime}\right) \equiv-e^{ \pm 2 i\left(\varphi_{\left.1-\mathbf{l}^{\prime}-\varphi_{1}\right)}\right.}\left[\gamma_{1}\left(\mathbf{l}-\mathbf{l}^{\prime}\right) \pm i \gamma_{2}\left(\mathbf{l}-\mathbf{l}^{\prime}\right)\right]+\int \frac{d^{2} \mathbf{l}^{\prime \prime}}{(2 \pi)^{2}} e^{ \pm 2 i\left(\varphi_{\left.1-\mathbf{l}^{\prime}-\varphi_{1}\right)}\right.}\left[\gamma_{1}\left(\mathbf{l}^{\prime \prime}\right) \pm i \gamma_{2}\left(\mathbf{l}^{\prime \prime}\right)\right] \times\left[\mathbf{l}^{\prime} \cdot\left(\mathbf{l}-\mathbf{l}^{\prime}-\mathbf{l}^{\prime \prime}\right)\right] \phi\left(\mathbf{l}-\mathbf{l}^{\prime}-\mathbf{l}^{\prime \prime}\right) \ldots
$$

Similarly for the local coupling systematics, the Taylor expansion of two Stokes polarization parameters including the systematic parameters can be written as

$$
\begin{aligned}
{[\tilde{Q} \pm i \tilde{U}]^{o b s}(\hat{\mathbf{n}})=} & (1+\delta)[Q \pm i U](\hat{\mathbf{n}}+\nabla \phi(\hat{\mathbf{n}}) ; \sigma)=[Q \pm i U](\hat{\mathbf{n}}+\nabla \phi(\hat{\mathbf{n}}) ; \sigma)+\sigma \mathbf{p}(\hat{\mathbf{n}}) \cdot \nabla[Q \pm i U](\hat{\mathbf{n}}+\nabla \phi(\hat{\mathbf{n}}) ; \sigma) \\
& +\sigma\left[d_{1} \pm i d_{2}\right](\hat{\mathbf{n}})\left[\partial_{1} \pm i \partial_{2}\right] T(\hat{\mathbf{n}}+\nabla \phi(\hat{\mathbf{n}}) ; \sigma)+\sigma^{2} q(\hat{\mathbf{n}})\left[\partial_{1} \pm i \partial_{2}\right]^{2} T(\hat{\mathbf{n}}+\nabla \phi(\hat{\mathbf{n}}) ; \sigma) \\
= & {\left.[Q \pm i U](\hat{\mathbf{n}} ; \sigma)+\nabla_{a} \phi(\hat{\mathbf{n}}) \nabla^{a}[Q \pm i U](\hat{\mathbf{n}} ; \sigma)+\frac{1}{2} \nabla_{a} \phi(\hat{\mathbf{n}}) \nabla_{b} \phi(\hat{\mathbf{n}}) \nabla^{a} \nabla^{b}[Q \pm i U](\hat{\mathbf{n}} ; \sigma)\right]+\ldots } \\
& +\sigma \mathbf{p}(\hat{\mathbf{n}}) \cdot \nabla\left[[Q \pm i U](\hat{\mathbf{n}} ; \sigma)+\nabla_{a} \phi(\hat{\mathbf{n}} ; \sigma) \nabla^{a}[Q \pm i U](\hat{\mathbf{n}} ; \sigma)\right. \\
& \left.+\frac{1}{2} \nabla_{a} \phi(\hat{\mathbf{n}} ; \sigma) \nabla_{b} \phi(\hat{\mathbf{n}} ; \sigma) \nabla^{a} \nabla^{b}[Q \pm i U](\hat{\mathbf{n}} ; \sigma)+\ldots\right] \\
& +\sigma\left[d_{1} \pm i d_{2}\right](\hat{\mathbf{n}})\left[\partial_{1} \pm i \partial_{2}\right]\left[T(\hat{\mathbf{n}} ; \sigma)+\nabla_{a} \phi(\hat{\mathbf{n}}) \nabla^{a} T(\hat{\mathbf{n}} ; \sigma)\right. \\
& \left.+\frac{1}{2} \nabla_{a} \phi(\hat{\mathbf{n}}) \nabla_{b} \phi(\hat{\mathbf{n}}) \nabla^{a} \nabla^{b} T(\hat{\mathbf{n}} ; \sigma)+\ldots\right] \\
& +\sigma^{2} q(\hat{\mathbf{n}})\left[\partial_{1} \pm i \partial_{2}\right]^{2}\left[T(\hat{\mathbf{n}} ; \sigma)+\nabla_{a} \phi(\hat{\mathbf{n}}) \nabla^{a} T(\hat{\mathbf{n}} ; \sigma)\right. \\
& \left.+\frac{1}{2} \nabla_{a} \phi(\hat{\mathbf{n}}) \nabla_{b} \phi(\hat{\mathbf{n}}) \nabla^{a} \nabla^{b} T(\hat{\mathbf{n}} ; \sigma)+\ldots\right] .
\end{aligned}
$$


Again performing the harmonic transformation gives

$$
\begin{aligned}
{[\tilde{E}(\mathbf{l}) \pm i \tilde{B}(\mathbf{l})]^{o b s} } & =\int d \hat{\mathbf{n}}[\tilde{Q} \pm i \tilde{U}]^{o b s}(\hat{\mathbf{n}}) e^{\mp 2 i \varphi \mathbf{l}} e^{-i \mathbf{l} \cdot \hat{\mathbf{n}}} \\
& =E(\mathbf{l}) \pm i B(\mathbf{l})-\int \frac{d^{2} \mathbf{l}^{\prime}}{(2 \pi)^{2}}\left[E\left(\mathbf{l}^{\prime}\right) \pm i B\left(\mathbf{l}^{\prime}\right)\right] M_{P}\left(\mathbf{l}, \mathbf{l}^{\prime}\right)-\int \frac{d^{2} \mathbf{l}^{\prime}}{(2 \pi)^{2}} T\left(\mathbf{l}^{\prime}\right) M_{T}\left(\mathbf{l}, \mathbf{l}^{\prime}\right)
\end{aligned}
$$

where

$$
\begin{aligned}
& M_{P}\left(\mathbf{l}, \mathbf{l}^{\prime}\right) \equiv e^{ \pm 2 i\left(\varphi_{\mathbf{l}^{\prime}}-\varphi_{1}\right)} \phi\left(\mathbf{l}-\mathbf{l}^{\prime}\right)\left[\left(\mathbf{l}-\mathbf{l}^{\prime}\right) \cdot \mathbf{l}^{\prime}\right]+\frac{1}{2} \int \frac{d^{2} \mathbf{l}^{\prime \prime}}{(2 \pi)^{2}} e^{ \pm 2 i\left(\varphi_{\mathbf{l}^{\prime}}-\varphi_{1}\right)} \phi\left(\mathbf{l}^{\prime \prime}\right) \phi\left(\mathbf{l}-\mathbf{l}^{\prime}-\mathbf{l}^{\prime \prime}\right)\left(\mathbf{l}^{\prime \prime} \cdot \mathbf{l}^{\prime}\right)\left[\left(\mathbf{l}^{\prime \prime}+\mathbf{l}^{\prime}-\mathbf{l}\right) \cdot \mathbf{l}^{\prime}\right]+\ldots \\
& \mp i \sigma \mathbf{l}^{\prime} \cdot\left[p_{a}\left(\mathbf{l}-\mathbf{l}^{\prime}\right) \pm i p_{b}\left(\mathbf{l}-\mathbf{l}^{\prime}\right)\right] e^{ \pm 2 i\left(\varphi_{1^{\prime}}-\varphi_{1}\right)} e^{ \pm i \varphi_{1-\mathbf{l}^{\prime}}} \\
& \pm i \sigma \int \frac{d^{2} \mathbf{l}^{\prime \prime}}{(2 \pi)^{2}}\left[p_{a}\left(\mathbf{l}^{\prime \prime}\right) \pm i p_{b}\left(\mathbf{l}^{\prime \prime}\right)\right] \cdot\left(\mathbf{l}-\mathbf{l}^{\prime \prime}\right)\left[\mathbf{l}^{\prime} \cdot\left(\mathbf{l}-\mathbf{l}^{\prime}-\mathbf{l}^{\prime \prime}\right)\right] \phi\left(\mathbf{l}-\mathbf{l}^{\prime}-\mathbf{l}^{\prime \prime}\right) e^{ \pm 2 i\left(\varphi_{\mathbf{1}^{\prime}}-\varphi_{1}\right)} e^{ \pm i \varphi_{\mathbf{l}^{\prime \prime}}} \ldots
\end{aligned}
$$

and

$$
\begin{aligned}
& M_{T}\left(\mathbf{l}, \mathbf{l}^{\prime}\right) \equiv i \sigma \mathbf{l}^{\prime}\left[d_{a}\left(\mathbf{l}-\mathbf{l}^{\prime}\right) \pm i d_{b}\left(\mathbf{l}-\mathbf{l}^{\prime}\right)\right] e^{ \pm i\left(\varphi_{\mathbf{I}^{\prime}}+\varphi_{\mathbf{1}-\mathbf{I}^{\prime}}-2 \varphi_{\mathbf{l}}\right)}
\end{aligned}
$$

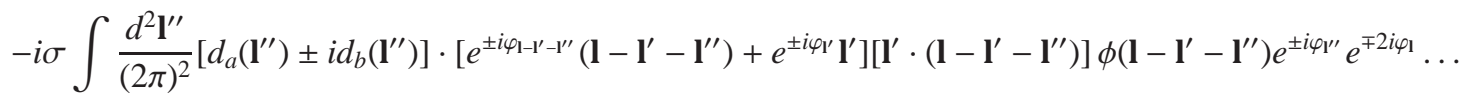

$$
\begin{aligned}
& +\sigma^{2} q\left(\mathbf{l}-\mathbf{l}^{\prime}\right) \mathbf{l}^{\prime 2} e^{ \pm 2 i\left(\varphi_{\mathbf{l}^{\prime}}-\varphi_{1}\right)} \\
& -\sigma^{2} \int \frac{d^{2} \mathbf{l}^{\prime \prime}}{(2 \pi)^{2}} q\left(\mathbf{l}^{\prime \prime}\right)\left[e^{ \pm 2 i\left(\varphi_{\left.1-\mathbf{l}^{\prime}-\mathbf{l}^{\prime \prime}-\varphi_{\mathbf{l}}\right)}\right.}\left(\mathbf{l}-\mathbf{l}^{\prime}-\mathbf{l}^{\prime \prime}\right)^{2}+e^{ \pm 2 i\left(\varphi_{\mathbf{l}^{\prime}}-\varphi_{1}\right)} \mathbf{1}^{\prime 2}\right]\left[\mathbf{l}^{\prime} \cdot\left(\mathbf{l}-\mathbf{l}^{\prime}-\mathbf{l}^{\prime \prime}\right)\right] \phi\left(\mathbf{l}-\mathbf{l}^{\prime}-\mathbf{l}^{\prime \prime}\right) \ldots
\end{aligned}
$$

Using equations (B2)-(B8), the window functions for each systematic fields (defined in Table difficulty.

\section{APPENDIX C: SYSTEMATIC CONTAMINATION IN OTHER ESTIMATORS}

In order to keep the paper short and clean, we focused on the EB estimator, one among six possible quadratic estimators of $\mathrm{CMB}$ fields. In this appendix, we give the results for EE, TE, and TB estimators.

\section{EE estimator}

For CMBPol like experiment, for multiple $l \lesssim 100$, EE estimator has comparable noise for the $C_{l}^{d d}$ reconstruction (especially in case of noise dominated B-mode detection). If one has good signal to noise ratio on E-mode detection, EE estimator can be a better choice compared to EB estimator. Here we calculate the systematic contamination for EE estimator. Again, we first calculate the connected trispectrum, which can be written in a compact form using the window functions defined in Table

$$
\begin{aligned}
\left\langle\tilde{E}\left(\mathbf{l}_{1}\right)^{\mathrm{obs}} \tilde{E}\left(\mathbf{l}_{2}\right)^{\mathrm{obs}} \tilde{E}\left(\mathbf{l}_{1}^{\prime}\right)^{\mathrm{obs}} \tilde{E}\left(\mathbf{l}_{2}^{\prime}\right)^{\mathrm{obs}}\right\rangle_{c}=(2 \pi)^{2} \delta_{\mathrm{D}}\left(\mathbf{l}_{1}+\mathbf{l}_{2}+\mathbf{l}_{1}^{\prime}+\mathbf{l}_{2}^{\prime}\right) \times & \\
& \left\{C_{l_{1}}^{E E} C_{l_{1}^{\prime}}^{E E}\left\langle\phi\left(\mathbf{l}_{1}+\mathbf{l}_{2}\right) \phi\left(\mathbf{l}_{1}^{\prime}+\mathbf{l}_{2}^{\prime}\right)\right\rangle W_{E}\left(\mathbf{l}_{2},-\mathbf{l}_{1}\right) W_{E}\left(\mathbf{l}_{2}^{\prime},-\mathbf{l}_{1}^{\prime}\right)\right. \\
& +\sum_{S S^{\prime}}^{P-\text { distortion }} C_{l_{1}}^{E E} C_{l_{1}^{\prime}}^{E E}\left\langle S\left(\mathbf{l}_{1}+\mathbf{l}_{2}\right) S^{\prime}\left(\mathbf{l}_{1}^{\prime}+\mathbf{l}_{2}^{\prime}\right)\right\rangle W_{E}^{S}\left(\mathbf{l}_{2},-\mathbf{l}_{1}\right) W_{E}^{S^{\prime}}\left(\mathbf{l}_{2}^{\prime},-\mathbf{l}_{1}^{\prime}\right) \\
& +\sum_{S S^{\prime}}^{T-\text { leakage }} C_{l_{1}}^{T E} C_{l_{1}^{\prime}}^{T E}\left\langle S\left(\mathbf{l}_{1}+\mathbf{l}_{2}\right) S^{\prime}\left(\mathbf{l}_{1}^{\prime}+\mathbf{l}_{2}^{\prime}\right)\right\rangle W_{E}^{S}\left(\mathbf{l}_{2},-\mathbf{l}_{1}\right) W_{E}^{S^{\prime}}\left(\mathbf{l}_{2}^{\prime},-\mathbf{l}_{1}^{\prime}\right) \\
& +C_{l_{1}}^{E E} C_{l_{1}^{\prime}}^{E E}\left\langle\phi\left(\mathbf{l}_{1}+\mathbf{l}_{2}^{\prime}\right) \phi\left(\mathbf{l}_{2}+\mathbf{l}_{1}^{\prime}\right)\right\rangle W_{E}\left(\mathbf{l}_{2},-\mathbf{l}_{1}^{\prime}\right) W_{E}\left(\mathbf{l}_{2}^{\prime},-\mathbf{l}_{1}\right) \\
& +\sum_{P-\text { distortion }} C_{l_{1}}^{E E} C_{l_{1}^{\prime}}^{E E}\left\langle S\left(\mathbf{l}_{1}+\mathbf{l}_{2}^{\prime}\right) S^{\prime}\left(\mathbf{l}_{2}+\mathbf{l}_{1}^{\prime}\right)\right\rangle W_{E}^{S}\left(\mathbf{l}_{2},-\mathbf{l}_{1}^{\prime}\right) W_{E}^{S^{\prime}}\left(\mathbf{l}_{2}^{\prime},-\mathbf{l}_{1}\right) \\
& +\sum_{S S^{\prime}}^{T-\text { leakage }} C_{l_{1}}^{T E} C_{l_{1}^{\prime}}^{T E}\left\langle S\left(\mathbf{l}_{1}+\mathbf{l}_{2}^{\prime}\right) S^{\prime}\left(\mathbf{l}_{2}+\mathbf{l}_{1}^{\prime}\right)\right\rangle W_{E}^{S}\left(\mathbf{l}_{2},-\mathbf{l}_{1}^{\prime}\right) W_{E}^{S^{\prime}}\left(\mathbf{l}_{2}^{\prime},-\mathbf{l}_{1}\right)+P e r m .
\end{aligned}
$$


Further averaging over the lensing and the systematic field gives

$$
\begin{aligned}
& \left\langle\tilde{E}\left(\mathbf{l}_{1}\right)^{\mathrm{obs}} \tilde{E}\left(\mathbf{l}_{2}\right)^{\mathrm{obs}} \tilde{E}\left(\mathbf{l}_{1}^{\prime}\right)^{\mathrm{obs}} \tilde{E}\left(\mathbf{l}_{2}^{\prime}\right)^{\mathrm{obs}}\right\rangle_{c}=(2 \pi)^{2} \delta_{\mathrm{D}}\left(\mathbf{l}_{1}+\mathbf{l}_{2}+\mathbf{l}_{1}^{\prime}+\mathbf{l}_{2}^{\prime}\right) \times \\
& \left\{C _ { l _ { 1 } } ^ { E E } C _ { l _ { 1 } ^ { \prime } } ^ { E E } \left[C_{\left|\mathbf{l}_{1}+\mathbf{l}_{2}\right|}^{\phi \phi} W_{E}\left(\mathbf{l}_{2},-\mathbf{l}_{1}\right) W_{E}\left(\mathbf{l}_{2}^{\prime},-\mathbf{l}_{1}^{\prime}\right)+C_{\left|\mathbf{l}_{1}+\mathbf{l}_{2}^{\prime}\right|}^{\phi \phi} W_{E}\left(\mathbf{l}_{2},-\mathbf{l}_{1}^{\prime}\right) W_{E}\left(\mathbf{l}_{2}^{\prime},-\mathbf{l}_{1}\right)\right.\right. \\
& \left.+\sum_{S}^{P \text {-distortion }} C_{\left|\mathbf{l}_{1}+\mathbf{l}_{2}\right|}^{S S} W_{E}^{S}\left(\mathbf{l}_{2},-\mathbf{l}_{1}\right) W_{E}^{S}\left(\mathbf{l}_{2}^{\prime},-\mathbf{l}_{1}^{\prime}\right)+\sum_{S}^{P \text {-distortion }} C_{\left|\mathbf{l}_{1}+\mathbf{l}_{2}\right|}^{S S} W_{E}^{S}\left(\mathbf{l}_{2},-\mathbf{l}_{1}^{\prime}\right) W_{E}^{S}\left(\mathbf{l}_{2}^{\prime},-\mathbf{l}_{1}\right)\right] \\
& +C_{l_{1}}^{T E} C_{l_{1}^{\prime}}^{T E}\left[\sum_{S}^{T \text {-leakage }} C_{\left|\mathbf{l}_{1}+\mathbf{l}_{2}\right|}^{S S} W_{E}^{S}\left(\mathbf{l}_{2},-\mathbf{l}_{1}\right) W_{E}^{S}\left(\mathbf{l}_{2}^{\prime},-\mathbf{l}_{1}^{\prime}\right)+\right. \\
& \left.\left.\sum_{S}^{\text {T-leakage }} C_{\left|\mathbf{I}_{1}+\mathbf{l}_{2}^{\prime}\right|}^{S S} W_{E}^{S}\left(\mathbf{l}_{2},-\mathbf{l}_{1}^{\prime}\right) W_{E}^{S}\left(\mathbf{l}_{2}^{\prime},-\mathbf{l}_{1}\right)\right]\right\}+ \text { Perm, }
\end{aligned}
$$

where we define the lensing E-mode window function as $W_{E}\left(\mathbf{l}, \mathbf{I}^{\prime}\right) \equiv-\mathbf{I}^{\prime} \cdot\left(\mathbf{l}-\mathbf{I}^{\prime}\right) \cos 2\left(\varphi_{\mathbf{I}^{\prime}}-\varphi_{1}\right)$. The systematic window functions $W_{E}^{S}\left(\mathbf{l}_{1},-\mathbf{l}_{1}^{\prime}\right)$ for any of those 11 systematics parameters are defined in Table II. Hence we obtain the CMB E-mode polarization trispectrum due to gravitational lensing in the presence of systematic contamination. We define

$$
f_{P}^{S}\left(\mathbf{l}_{1}, \mathbf{l}_{2}\right)=C_{l_{1}}^{E E} W_{E}^{S}\left(\mathbf{l}_{1}, \mathbf{l}_{2}\right)+C_{l_{2}}^{E E} W_{E}^{S}\left(\mathbf{l}_{2}, \mathbf{l}_{1}\right)
$$

and

$$
f_{T}^{S}\left(\mathbf{l}_{1}, \mathbf{l}_{2}\right)=C_{l_{1}}^{T E} W_{E}^{S}\left(\mathbf{l}_{1}, \mathbf{l}_{2}\right)+C_{l_{2}}^{T E} W_{E}^{S}\left(\mathbf{l}_{2}, \mathbf{l}_{1}\right)
$$

Thus Eq. (C2) could be written as

$$
\begin{aligned}
& \left\langle\tilde{E}\left(\mathbf{l}_{1}\right)^{\mathrm{obs}} \tilde{E}\left(\mathbf{l}_{2}\right)^{\mathrm{obs}} \tilde{E}\left(\mathbf{l}_{1}^{\prime}\right)^{\mathrm{obs}} \tilde{E}\left(\mathbf{l}_{2}^{\prime}\right)^{\mathrm{obs}}\right\rangle_{c}=\left[C_{\left|\mathbf{l}_{1}+\mathbf{l}_{2}\right|}^{\phi \phi} f_{E E}\left(\mathbf{l}_{1}, \mathbf{l}_{2}\right) f_{E E}\left(\mathbf{l}_{1}{ }^{\prime}, \mathbf{l}_{2}{ }^{\prime}\right)+C_{\left|\mathbf{l}_{1}+\mathbf{l}_{1}{ }^{\prime}\right|}^{\phi \phi} f_{E E}\left(\mathbf{l}_{1}, \mathbf{l}_{1}{ }^{\prime}\right) f_{E E}\left(\mathbf{l}_{2}, \mathbf{l}_{2}{ }^{\prime}\right)+C_{\left|\mathbf{l}_{1}+\mathbf{l}_{2}{ }^{\prime}\right|}^{\phi \phi} f_{E E}\left(\mathbf{l}_{1}, \mathbf{l}_{2}{ }^{\prime}\right) f_{E E}\left(\mathbf{l}_{2}, \mathbf{l}_{1}{ }^{\prime}\right)\right] \\
& +\sum_{S S^{\prime}}^{P-\text { distortion }}\left[C_{\left|\mathbf{l}_{1}+\mathbf{l}_{2}\right|}^{S S} f_{E E}^{S}\left(\mathbf{l}, \mathbf{l}^{\prime}\right) f_{E E}^{S}\left(\mathbf{l}, \mathbf{l}^{\prime}\right)+C_{\left|\mathbf{l}_{1}+\mathbf{l}_{1}{ }^{\prime}\right|}^{S S} f_{E E}^{S}\left(\mathbf{l}_{1}, \mathbf{l}_{1}{ }^{\prime}\right) f_{E E}^{S}\left(\mathbf{l}_{2}, \mathbf{l}_{2}{ }^{\prime}\right)+C_{\left|\mathbf{l}_{1}+\mathbf{l}_{2}{ }^{\prime}\right|}^{S S} f_{E E}^{S}\left(\mathbf{l}_{1}, \mathbf{l}_{2}{ }^{\prime}\right) f_{E E}^{S}\left(\mathbf{l}_{2}, \mathbf{l}_{1}{ }^{\prime}\right)\right] \\
& +\sum_{S S^{\prime}}^{T \text {-leakage }}\left[C_{\left|\mathbf{l}_{1}+\mathbf{l}_{2}\right|}^{S S} f_{E E}^{S}\left(\mathbf{l}, \mathbf{l}^{\prime}\right) f_{E E}^{S}\left(\mathbf{l}, \mathbf{l}^{\prime}\right)+C_{\left|\mathbf{l}_{1}+\mathbf{l}_{1}{ }^{\prime}\right|}^{S S} f_{E E}^{S}\left(\mathbf{l}_{1}, \mathbf{l}_{1}{ }^{\prime}\right) f_{E E}^{S}\left(\mathbf{l}_{2}, \mathbf{l}_{2}{ }^{\prime}\right)+C_{\left|\mathbf{l}_{1}+\mathbf{l}_{2}\right|}^{S S} f_{E E}^{S}\left(\mathbf{l}_{1}, \mathbf{l}_{2}{ }^{\prime}\right) f_{E E}^{S}\left(\mathbf{l}_{2}, \mathbf{l}_{1}{ }^{\prime}\right)\right] \text {, }
\end{aligned}
$$

where the permutations now contain 5 additional terms with the replacement of $\left(\mathbf{l}_{3}, \mathbf{l}_{4}\right)$ pair by other combination of pairs $\left(\mathbf{l}_{1}, \mathbf{l}_{2}\right)$, $\left(\mathbf{I}_{1}, \mathbf{l}_{3}\right),\left(\mathbf{l}_{1}, \mathbf{l}_{4}\right),\left(\mathbf{l}_{2}, \mathbf{l}_{3}\right),\left(\mathbf{l}_{2}, \mathbf{l}_{4}\right)$. One can simply Plug in the trispectrum given above to the deflection angle power spectrum estimation (the final result can be written as a sum of deflection angle power spectrum and noise terms)

$$
\begin{aligned}
\left\langle\left\langle\left\langle d_{E E}(\mathbf{L}) \cdot d_{E E}\left(\mathbf{L}^{\prime}\right)\right\rangle_{\mathrm{CMB}}\right\rangle_{\mathrm{LSS}}\right\rangle_{\mathrm{SYS}}= & \frac{A_{E E}(L)}{L^{2}} \frac{A_{E E}\left(L^{\prime}\right)}{L^{\prime 2}} \int \frac{d^{2} \mathbf{l}_{1}}{(2 \pi)^{2}} \int \frac{d^{2} \mathbf{l}_{1}^{\prime}}{(2 \pi)^{2}} F_{E E}\left(\mathbf{l}_{1}, \mathbf{l}_{2}\right) F_{E E}\left(\mathbf{l}_{1}{ }^{\prime}, \mathbf{l}_{2}{ }^{\prime}\right) \\
& \times\left\langle\tilde{E}\left(\mathbf{l}_{1}\right)^{\mathrm{obs}} \tilde{E}\left(\mathbf{l}_{2}\right)^{\mathrm{obs}} \tilde{E}\left(\mathbf{l}_{1}^{\prime}\right)^{\mathrm{obs}} \tilde{E}\left(\mathbf{l}_{2}^{\prime}\right)^{\mathrm{obs}}\right\rangle \\
= & (2 \pi)^{2} \delta_{\mathrm{D}}\left(\mathbf{L}+\mathbf{L}^{\prime}\right)\left[C^{d d}(L)+N_{E E, E E}^{(0)}(L)+N_{E E, E E}^{(1)}(L)+N_{E E, E E}^{(S)}(L)+\ldots\right],
\end{aligned}
$$

where $\mathbf{L}=\mathbf{l}_{1}+\mathbf{l}_{2}$, and $C^{d d}(L)$ is the deflection angle power spectrum. The terms $N_{E E, E E}^{(0)}(L), N_{E E, E E}^{(1)}(L)$ and $N_{E E, E E}^{(S)}(L)$ are the Gaussian noise, first order non-Gaussian noise, and the first order systematics noise for the EE estimator respectively. The systematics noise term $N_{E E, E E}^{(S)}(L)$ can be written as

$$
\begin{aligned}
& N_{E E, E E}^{(S)}(L)=\frac{A_{E E}^{2}(L)}{L^{2}} \int \frac{d^{2} \mathbf{l}_{1}}{(2 \pi)^{2}} \int \frac{d^{2} \mathbf{l}_{1}^{\prime}}{(2 \pi)^{2}} F_{E E}\left(\mathbf{l}_{1}, \mathbf{l}_{2}\right) F_{E E}\left(\mathbf{l}_{1}{ }^{\prime}, \mathbf{l}_{2}{ }^{\prime}\right) \\
&\left\{C_{l_{1}}^{E E} C_{l_{1}^{\prime}}^{E E}\left[\sum_{S}^{P \text {-distortion }} C_{\left|\mathbf{l}_{1}+\mathbf{l}_{2}\right|}^{S S} W_{E}^{S}\left(\mathbf{l}_{2},-\mathbf{l}_{1}\right) W_{E}^{S}\left(\mathbf{l}_{2}^{\prime},-\mathbf{l}_{1}^{\prime}\right)+\sum_{S}^{P \text {-distortion }} C_{\left|\mathbf{l}_{1}+\mathbf{l}_{2}^{\prime}\right|}^{S S} W_{E}^{S}\left(\mathbf{l}_{2},-\mathbf{l}_{1}^{\prime}\right) W_{E}^{S}\left(\mathbf{l}_{2}^{\prime},-\mathbf{l}_{1}\right)\right]\right. \\
&\left.+C_{l_{1}}^{T E} C_{l_{1}^{\prime}}^{T E}\left[, \sum_{S}^{T-\text { leakage }} C_{\left|\mathbf{l}_{1}+\mathbf{l}_{2}\right|}^{S S} W_{E}^{S}\left(\mathbf{l}_{2},-\mathbf{l}_{1}\right) W_{E}^{S}\left(\mathbf{l}_{2}^{\prime},-\mathbf{l}_{1}^{\prime}\right)+\sum_{S}^{T \text {-leakage }} C_{\left|\mathbf{l}_{1} \mathbf{l}_{\mathbf{l}^{\prime}}\right|^{\prime}}^{S S} W_{E}^{S}\left(\mathbf{l}_{2},-\mathbf{l}_{1}^{\prime}\right) W_{E}^{S}\left(\mathbf{l}_{2}^{\prime},-\mathbf{l}_{1}\right)\right]\right\},
\end{aligned}
$$

where the systematics window functions $W_{E}^{S}\left(\mathbf{l}_{1}, \mathbf{l}_{2}\right)$ are given in Table $\Pi$ 


\section{TB and TE estimator}

The instrumental systematic contamination calculation for the TB and TE estimator is similar to the calculation of EB estimator. At leading order of $C_{l}^{\phi \phi}$ and $C_{l}^{S S}$, we have

$$
\begin{aligned}
& \left\langle\tilde{T}\left(\mathbf{l}_{1}\right)^{\mathrm{obs}} \tilde{X}\left(\mathbf{l}_{2}\right)^{\mathrm{obs}} \tilde{T}\left(\mathbf{l}_{1}^{\prime}\right)^{\mathrm{obs}} \tilde{X}\left(\mathbf{l}_{2}^{\prime}\right)^{\mathrm{obs}}\right\rangle_{c}=(2 \pi)^{2} \delta_{\mathrm{D}}\left(\mathbf{l}_{1}+\mathbf{l}_{2}+\mathbf{l}_{1}^{\prime}+\mathbf{l}_{2}^{\prime}\right) \times \\
& \left\{C_{l_{1}}^{T E} C_{l_{1}^{\prime}}^{T E}\left\langle\phi\left(\mathbf{l}_{1}+\mathbf{l}_{2}\right) \phi\left(\mathbf{l}_{1}^{\prime}+\mathbf{l}_{2}^{\prime}\right)\right\rangle W_{X}\left(\mathbf{l}_{2},-\mathbf{l}_{1}\right) W_{X}\left(\mathbf{l}_{2}^{\prime},-\mathbf{l}_{1}^{\prime}\right)\right. \\
& +\sum_{S S^{\prime}}^{P-\text { distortion }} C_{l_{1}}^{T E} C_{l_{1}^{\prime}}^{T E}\left\langle S\left(\mathbf{l}_{1}+\mathbf{l}_{2}\right) S^{\prime}\left(\mathbf{l}_{1}^{\prime}+\mathbf{l}_{2}^{\prime}\right)\right\rangle W_{X}^{S}\left(\mathbf{l}_{2},-\mathbf{l}_{1}\right) W_{X}^{S^{\prime}}\left(\mathbf{l}_{2}^{\prime},-\mathbf{l}_{1}^{\prime}\right) \\
& +\sum_{S S^{\prime}}^{T \text {-leakage }} C_{l_{1}}^{T T} C_{l_{1}^{\prime}}^{T T}\left\langle S\left(\mathbf{l}_{1}+\mathbf{l}_{2}\right) S^{\prime}\left(\mathbf{l}_{1}^{\prime}+\mathbf{l}_{2}^{\prime}\right)\right\rangle W_{X}^{S}\left(\mathbf{l}_{2},-\mathbf{l}_{1}\right) W_{X}^{S^{\prime}}\left(\mathbf{l}_{2}^{\prime},-\mathbf{l}_{1}^{\prime}\right) \\
& +C_{l_{1}}^{T T} C_{l_{1}^{\prime}}^{T T}\left\langle\phi\left(\mathbf{l}_{1}+\mathbf{l}_{2}^{\prime}\right) \phi\left(\mathbf{l}_{2}+\mathbf{l}_{1}^{\prime}\right)\right\rangle W_{X}\left(\mathbf{l}_{2},-\mathbf{l}_{1}^{\prime}\right) W_{X}\left(\mathbf{l}_{2}^{\prime},-\mathbf{l}_{1}\right) \\
& +\sum_{S S^{\prime}}^{P-\text { distortion }} C_{l_{1}}^{T T} C_{l_{1}^{\prime}}^{T T}\left\langle S\left(\mathbf{l}_{1}+\mathbf{l}_{2}^{\prime}\right) S^{\prime}\left(\mathbf{l}_{2}+\mathbf{l}_{1}^{\prime}\right)\right\rangle W_{X}^{S}\left(\mathbf{l}_{2},-\mathbf{l}_{1}^{\prime}\right) W_{X}^{S^{\prime}}\left(\mathbf{l}_{2}^{\prime},-\mathbf{l}_{1}\right) \\
& \left.+\sum_{S S^{\prime}}^{T \text {-leakage }} C_{l_{1}}^{T E} C_{l_{1}^{\prime}}^{T E}\left\langle S\left(\mathbf{l}_{1}+\mathbf{l}_{2}^{\prime}\right) S^{\prime}\left(\mathbf{l}_{2}+\mathbf{l}_{1}^{\prime}\right)\right\rangle W_{X}^{S}\left(\mathbf{l}_{2},-\mathbf{l}_{1}^{\prime}\right) W_{X}^{S^{\prime}}\left(\mathbf{l}_{2}^{\prime},-\mathbf{l}_{1}\right)\right\} \\
& =(2 \pi)^{2} \delta_{\mathrm{D}}\left(\mathbf{l}_{1}+\mathbf{l}_{2}+\mathbf{l}_{1}^{\prime}+\mathbf{l}_{2}^{\prime}\right) \times \\
& \left\{C _ { l _ { 1 } } ^ { T E } C _ { l _ { 1 } ^ { \prime } } ^ { T E } \left[C_{\left|\mathbf{l}_{1}+\mathbf{l}_{2}\right|}^{\phi \phi} W_{X}\left(\mathbf{l}_{2},-\mathbf{l}_{1}\right) W_{X}\left(\mathbf{l}_{2}^{\prime},-\mathbf{l}_{1}^{\prime}\right)+C_{\left|\mathbf{l}_{1}+\mathbf{l}_{2}^{\prime}\right|}^{\phi \phi} W_{X}\left(\mathbf{l}_{2},-\mathbf{l}_{1}^{\prime}\right) W_{X}\left(\mathbf{l}_{2}^{\prime},-\mathbf{l}_{1}\right)\right.\right. \\
& \left.+\sum_{S}^{P \text {-distortion }} C_{\left|\mathbf{l}_{1}+\mathbf{l}_{2}\right|}^{S S} W_{X}^{S}\left(\mathbf{l}_{2},-\mathbf{l}_{1}\right) W_{X}^{S}\left(\mathbf{l}_{2}^{\prime},-\mathbf{l}_{1}^{\prime}\right)+\sum_{S}^{P-\text { distortion }} C_{\left|\mathbf{l}_{1}+\mathbf{l}_{2}^{\prime}\right|}^{S S} W_{X}^{S}\left(\mathbf{l}_{2},-\mathbf{l}_{1}^{\prime}\right) W_{X}^{S}\left(\mathbf{l}_{2}^{\prime},-\mathbf{l}_{1}\right)\right] \\
& +C_{l_{1}}^{T T} C_{l_{1}^{\prime}}^{T T}\left[\sum_{S}^{T \text {-leakage }} C_{\left|\mathbf{l}_{1}+\mathbf{l}_{2}\right|}^{S S} W_{X}^{S}\left(\mathbf{l}_{2},-\mathbf{l}_{1}\right) W_{X}^{S}\left(\mathbf{l}_{2}^{\prime},-\mathbf{l}_{1}^{\prime}\right)+\right. \\
& \left.\left.\sum_{S}^{\text {T-leakage }} C_{\left|\mathbf{l}_{1}+\mathbf{l}_{2}\right|}^{S S} W_{X}^{S}\left(\mathbf{l}_{2},-\mathbf{l}_{1}^{\prime}\right) W_{X}^{S}\left(\mathbf{l}_{2}^{\prime},-\mathbf{l}_{1}\right)\right]\right\}
\end{aligned}
$$

where $X=E$ for the $T E$ estimator, and $X=B$ for the $T B$ estimator. We define the lensing window function $W_{B}\left(\mathbf{l}, \mathbf{l}^{\prime}\right) \equiv$ $\mathbf{l}^{\prime} \cdot\left(\mathbf{l}-\mathbf{l}^{\prime}\right) \sin 2\left(\varphi_{\mathbf{l}}-\varphi_{\mathbf{I}^{\prime}}\right)$ and $W_{E}\left(\mathbf{l}, \mathbf{l}^{\prime}\right) \equiv-\mathbf{l}^{\prime} \cdot\left(\mathbf{l}-\mathbf{l}^{\prime}\right) \cos 2\left(\varphi_{\mathbf{l}}-\varphi_{\mathbf{I}^{\prime}}\right)$. Window $W_{E}^{S}\left(\mathbf{l}_{1},-\mathbf{l}_{1}^{\prime}\right)$ is the systematics window function for any of those 11 systematics parameters we defined in Table $\amalg$ One can simply Plug in the trispectrum given above to the deflection angle power spectrum estimation (the final result can be written as a sum of deflection angle power spectrum and noise terms),

$$
\begin{aligned}
\left\langle\left\langle\left\langle d_{T X}(\mathbf{L}) \cdot d_{T X}\left(\mathbf{L}^{\prime}\right)\right\rangle_{\mathrm{CMB}}\right\rangle_{\mathrm{LSS}}\right\rangle_{\mathrm{SYS}}= & \frac{A_{T X}(L)}{L^{2}} \frac{A_{T X}\left(L^{\prime}\right)}{L^{\prime 2}} \int \frac{d^{2} \mathbf{l}_{1}}{(2 \pi)^{2}} \int \frac{d^{2} \mathbf{l}_{1}^{\prime}}{(2 \pi)^{2}} F_{T X}\left(\mathbf{l}_{1}, \mathbf{l}_{2}\right) F_{T X}\left(\mathbf{l}_{1}{ }^{\prime}, \mathbf{l}_{2}{ }^{\prime}\right) \\
& \times\left\langle\tilde{T}\left(\mathbf{l}_{1}\right)^{\mathrm{obs}} \tilde{X}\left(\mathbf{l}_{2}\right)^{\mathrm{obs}} \tilde{T}\left(\mathbf{l}_{1}^{\prime}\right)^{\mathrm{obs}} \tilde{X}\left(\mathbf{l}_{2}^{\prime}\right)^{\mathrm{obs}}\right\rangle \\
= & (2 \pi)^{2} \delta_{\mathrm{D}}\left(\mathbf{L}+\mathbf{L}^{\prime}\right)\left[C^{d d}(L)+N_{T X, T X}^{(0)}(L)+N_{T X, T X}^{(1)}(L)+N_{T X, T X}^{(S)}(L)+\ldots\right]
\end{aligned}
$$

where $\mathbf{L}=\mathbf{l}_{1}+\mathbf{l}_{2}$, and $C^{d d}(L)$ is the deflection angle power spectrum. The terms $N_{T X, T X}^{(0)}(L), N_{T X, T X}^{(1)}(L)$ and $N_{T X, T X}^{(S)}(L)$ are the Gaussian noise, first order non-Gaussian noise, and the first order systematics noise for the TB/TE estimator respectively. The 
systematics noise term $N_{T X, T X}^{(S)}(L)$ can be written as

$$
\begin{aligned}
N_{T X, T X}^{(S)}(L)= & \frac{A_{T X}^{2}(L)}{L^{2}} \int \frac{d^{2} \mathbf{l}_{1}}{(2 \pi)^{2}} \int \frac{d^{2} \mathbf{l}_{1}^{\prime}}{(2 \pi)^{2}} F_{T X}\left(\mathbf{l}_{1}, \mathbf{l}_{2}\right) F_{T X}\left(\mathbf{l}_{1}{ }^{\prime}, \mathbf{l}_{2}{ }^{\prime}\right) \\
& \left\{C_{l_{1}}^{T X} C_{l_{1}^{\prime}}^{T X}\left[\sum_{S}^{P-\text { distortion }} C_{\left|\mathbf{l}_{1}+\mathbf{l}_{2}\right|}^{S S} W_{X}^{S}\left(\mathbf{l}_{2},-\mathbf{l}_{1}\right) W_{X}^{S}\left(\mathbf{l}_{2}^{\prime},-\mathbf{l}_{1}^{\prime}\right)+\sum_{S}^{P \text {-distortion }} C_{\left|\mathbf{l}_{1}+\mathbf{l}_{2}^{\prime}\right|}^{S S} W_{X}^{S}\left(\mathbf{l}_{2},-\mathbf{l}_{1}^{\prime}\right) W_{X}^{S}\left(\mathbf{l}_{2}^{\prime},-\mathbf{l}_{1}\right)\right]\right. \\
& \left.+C_{l_{1}}^{T T} C_{l_{1}^{\prime}}^{T T}\left[\sum_{S}^{T-\text { leakage }} C_{\left|\mathbf{l}_{1}+\mathbf{l}_{2}\right|}^{S S} W_{X}^{S}\left(\mathbf{l}_{2},-\mathbf{l}_{1}\right) W_{X}^{S}\left(\mathbf{l}_{2}^{\prime},-\mathbf{l}_{1}^{\prime}\right)+\sum_{S}^{T-\text { leakage }} C_{\left|\mathbf{l}_{1}+\mathbf{l}_{2}^{\prime}\right|}^{S S} W_{X}^{S}\left(\mathbf{l}_{2},-\mathbf{l}_{1}^{\prime}\right) W_{X}^{S}\left(\mathbf{l}_{2}^{\prime},-\mathbf{l}_{1}\right)\right]\right\},
\end{aligned}
$$

where the systematics window functions $W_{X}^{S}\left(\mathbf{l}_{1}, \mathbf{l}_{2}\right)$ are given in Table $\Pi$ 